\title{
Mathematical Analysis of a Cholera Model with Vaccination
}

\author{
Jing'an Cui, ${ }^{1}$ Zhanmin $\mathrm{Wu}{ }^{1}$ and Xueyong Zhou ${ }^{2}$ \\ ${ }^{1}$ School of Science, Beijing University of Civil Engineering and Architecture, Beijing 100044, China \\ ${ }^{2}$ College of Mathematics and Information Science, Xinyang Normal University, Xinyang, Henan 464000, China
}

Correspondence should be addressed to Jing’an Cui; cuijingan@bucea.edu.cn

Received 14 March 2013; Revised 18 May 2013; Accepted 26 September 2013; Published 13 February 2014

Academic Editor: Tin-Tai Chow

Copyright (C) 2014 Jing'an Cui et al. This is an open access article distributed under the Creative Commons Attribution License, which permits unrestricted use, distribution, and reproduction in any medium, provided the original work is properly cited.

\begin{abstract}
We consider a $S V R-B$ cholera model with imperfect vaccination. By analyzing the corresponding characteristic equations, the local stability of a disease-free equilibrium and an endemic equilibrium is established. We calculate the certain threshold known as the control reproduction number $\mathscr{R}_{v}$. If $\mathscr{R}_{v}<1$, we obtain sufficient conditions for the global asymptotic stability of the disease-free equilibrium; the diseases will be eliminated from the community. By comparison of arguments, it is proved that if $\mathscr{R}_{v}>1$, the disease persists and the unique endemic equilibrium is globally asymptotically stable, which is obtained by the second compound matrix techniques and autonomous convergence theorems. We perform sensitivity analysis of $\mathscr{R}_{v}$ on the parameters in order to determine their relative importance to disease transmission and show that an imperfect vaccine is always beneficial in reducing disease spread within the community.
\end{abstract}

\section{Introduction}

Cholera is an acute intestinal infection caused by the ingestion of food or water contaminated with the bacterium Vibrio cholera. Among the 200 serogroups of Vibrio cholera, it is only Vibrio cholera o1 and o139 that are known to be the cause of the cholera disease [1]. The etiological agent, Vibrio cholera ol (and more recently vibrio cholera o139), passes through and survives the gastric acid barrier of the stomach and then penetrates the mucus lining that coats the intestinal epithelial [2]. Once they colonise the intestinal gut, then produce enterotoxin (which stimulates water and electrolyte secretion by the endothelial cells of the small intestine) that leads to copious, painless, and watery diarrhoea that can quickly lead to severe dehydration and death if treatment is not promptly given. Vomiting also occurs in most patients. In human volunteer studies, the infection was determined to be $10^{2}-10^{3}$ [3]. Cholera can either be transmitted through interaction between humans (i.e., fecal-oral) or through interaction between humans and their environment (i.e., ingestion of contaminated water and food from the environment). To come on urgent, transmission fast, sweeping range widely are the characteristics of cholera which is one of the international quarantine infectious diseases as stipulated by the International Health Regulations (IHR), as well as one of a class of infectious diseases as stipulated by law for infectious diseases prevention and control of China.

Globally, cholera incidence has increased steadily since 2005 with cholera outbreaks affecting several continents (see Figure 1). Cholera continues to pose a serious public health problem among developing world populations which have no access to adequate water and sanitation resources. In 2011, 32\% of cases were reported from Africa whereas between 2001 and $2009,93 \%$ to $98 \%$ of total cases worldwide were reported from that continent [4]. In 2011, 61.2\% of cases were reported from Americas where a large outbreak that started in Haiti at the end of October 2010 also affected the Dominican Republic. The outbreak was still ongoing at the end of 2011 with 522335 cases including 7001 deaths that were reported by 25 December in Haiti [4]. So, cholera remains a global threat and is one of the key indicators of social development. The history and reality have warned: we are facing the serious threat of cholera, the importance for the study of cholera's pathogenesis, regular transmission and prevention and control strategy have become increasingly prominent, which has also become a major problem that needs to be solved. Up till now, a number of mathematical models have been used to study the transmission dynamics 


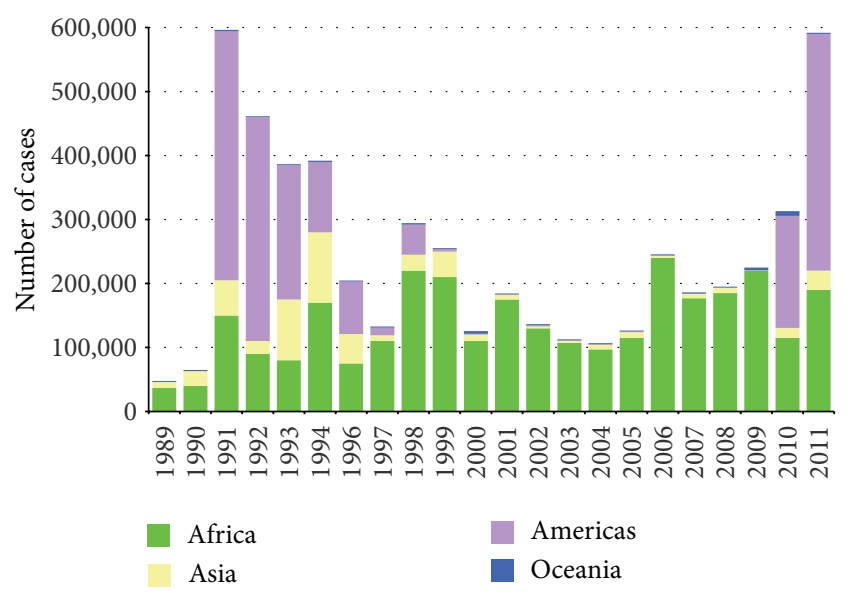

FIGURE 1: In 1989-2011, cholera cases were reported to World Health Organization by year and by continent [4].

of cholera. Capasso and Serio [5] introduced an incidence rate in the form of $k S I /(1+\alpha I)$ (with human-to-human transmission model only) in 1973 [6]. Codeço [7] proposed an incidence form of $a S B /(K+B)$ (with environment-tohuman transmission model only) in 2001 which, in the first time, explicitly incorporated the pathogen concentration into cholera modeling. Mukandavire et al. [8] included both transmission pathways in the form of $\beta_{h} S I+\left(\beta_{e} S B /(K+\right.$ $B)$ ). In 2012, Liao and Wang [9] generalised Codeco's model [7], incorporating the theory of Volterra-Lyapunov stable matrices into the classic method of Lyapunov functions; they studied the global dynamics of the mathematical model.

Vaccination is a major factor in the resurgence and epidemic outbreaks of some infectious diseases. Since the pioneering work of Edward Jenner on smallpox [10], vaccination has been a commonly used method for diseases control [11-13] and works by reducing the number of susceptible individuals in a population. In modern times, vaccination has a large impact on the incidence and persistence of children infections, such as measles and whooping cough [14]. Hethcote [11] investigated a pertussis infectious model and showed that the vaccination can make the infection undergo fluctuation. Although vaccination offers a very powerful tool for disease control, generally, vaccines are not $100 \%$ affective and sometimes they only provide limited immunity due to the natural waning of immunity in the host or antigenic variation in the pathogen [15].

Here, we develop a cholera model with an additional equation for the vaccinated individuals in the population. Since Koch found Vibrio cholera in 1883, the research for cholera vaccine had been going on for over one hundred years. People have developed a variety of vaccines. However, these vaccines were parenteral, which have short effective protection and big side effects. In 1973, the World Health Organization canceled the vaccine inoculation which attracted a major concern to oral vaccines. At present, there are three kinds of oral vaccines (i.e., WC/BS vaccine, $\mathrm{WC} / \mathrm{rBS}$ vaccine, and $\mathrm{CVD}_{103}-\mathrm{HgR}$ vaccine) have been proved to be safe, effective, and immunogenic, which were approved to apply in some countries [16].

In this paper, according to the natural history of cholera, we improve the model of [9] in the following two aspects. Firstly, if the cholera persists for a long time, it will cause death [17], especially in the area where water and sanitation resources are not adequate [4]; a parameter $d$ is added to describe the rate of disease-related death. Secondly, we propose a proportion of the vaccination in susceptible individuals as shown in the following differential equations:

$$
\begin{gathered}
\frac{d S(t)}{d t}=\mu_{1}-\frac{\beta_{1} S(t) B(t)}{1+\alpha_{1} B(t)}-\frac{\beta_{2} S(t) I(t)}{1+\alpha_{2} I(t)} \\
-\phi S(t)-\mu_{1} S(t)+\theta V(t), \\
\frac{d V(t)}{d t}=\phi S(t)-\theta V(t)-\mu_{1} V(t), \\
\frac{d I(t)}{d t}=\frac{\beta_{1} S(t) B(t)}{1+\alpha_{1} B(t)}+\frac{\beta_{2} S(t) I(t)}{1+\alpha_{2} I(t)}-\left(d+\alpha+\mu_{1}\right) I(t), \\
\frac{d R(t)}{d t}=\alpha I(t)-\mu_{1} R(t), \\
\frac{d B(t)}{d t}=\eta I(t)-\mu_{2} B(t) .
\end{gathered}
$$

The flow diagram of the model is depicted in Figure 2. Since the first three and last equations in (1) are independent of the variable $R$, it suffices to consider the following reduced model:

$$
\begin{gathered}
\frac{d S(t)}{d t}=\mu_{1}-\frac{\beta_{1} S(t) B(t)}{1+\alpha_{1} B(t)}-\frac{\beta_{2} S(t) I(t)}{1+\alpha_{2} I(t)}-\phi S(t) \\
-\mu_{1} S(t)+\theta V(t), \\
\frac{d V(t)}{d t}=\phi S(t)-\theta V(t)-\mu_{1} V(t), \\
\frac{d I(t)}{d t}=\frac{\beta_{1} S(t) B(t)}{1+\alpha_{1} B(t)}+\frac{\beta_{2} S(t) I(t)}{1+\alpha_{2} I(t)}-\left(d+\alpha+\mu_{1}\right) I(t), \\
\frac{d B(t)}{d t}=\eta I(t)-\mu_{2} B(t) .
\end{gathered}
$$

Here, $S, I, V$, and $R$ refer to the susceptible individuals, infected individuals, vaccinated individuals, and recovered individuals, respectively. The pathogen population at time $t$ is given by $B(t)$. The parameter $\mu_{1}$ denotes the natural human birth and death rate, $\alpha$ denotes the rate of recovery from the disease, $\eta$ represents the rate of human contribution to the growth of the pathogen, and $\mu_{2}$ represents the death rate of the pathogen in the environment. The coefficients $\beta_{1}$ and $\beta_{2}$ represent the contact rates for the human-environment and human-human interactions, respectively. Constants $\alpha_{1}$ and $\alpha_{2}$ adjust the appropriate form of the incidence which determines the rate of new infection. If $\alpha_{2}=0$, the corresponding incidence is reduced to the standard bilinear 


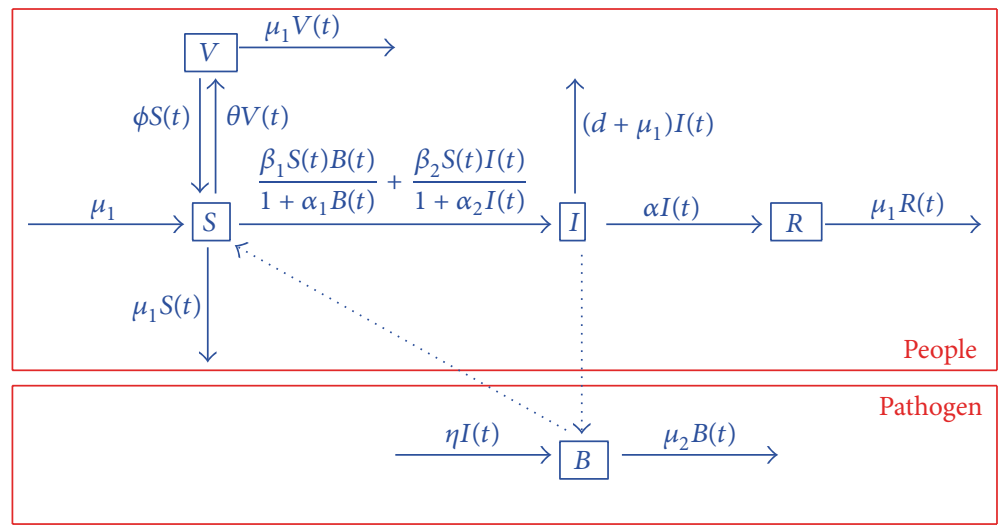

FIGURE 2: Progression of infection from susceptible $(S)$ and vaccinated $(V)$ individuals through the infected $(I)$ and recovered $(R)$ compartments for the combined human-environment epidemiological model with an environmental component.

form based on the mass action law, which is most common in epidemiological models. If $\alpha_{2}>0$, then the corresponding incidence represents a consequence of saturation effects: when the infected number is high, the incidence rate will respond more slowly than linearly to the increase in $I$. Similar meanings stand for $\alpha_{1}$. The rate at which the susceptible population is vaccinated is $\phi$, and the rate at which the vaccine wears off is $\theta$. All parameters are assumed nonnegative.

The organization of this paper is as follows: the positivity and boundedness of solutions are obtained in Section 2. In Section 3, we obtain the existence of the endemic equilibrium. We get the local and global stability of the diseasefree equilibrium in Section 4. In Section 5, we present the persistence of the system. In Section 6, we show the local and global stability of the endemic equilibrium. We analyze the sensitivity of $\mathscr{R}_{v}$ on the parameters, and we present the numerical simulation in Section 7 . The paper ends with a conclusion in Section 8.

\section{Positivity and Boundedness of Solutions}

In the following, we show that the solutions of system (2) are positive with the nonnegative initial conditions.

Theorem 1. The solutions $(S(t), V(t), I(t), B(t))$ of model (2) are nonnegative for all $t>0$ with the non-negative initial conditions.

Proof. System (2) can be put into the matrix form

$$
X^{\prime}=M(X)
$$

where $X=(S, V, I, B)^{T} \in R^{4}$ and $M(X)$ is given by

$$
M(X)=\left(\begin{array}{c}
M_{1}(X) \\
M_{2}(X) \\
M_{3}(X) \\
M_{4}(X)
\end{array}\right)
$$

$$
=\left(\begin{array}{c}
\mu_{1}-\frac{\beta_{1} S B}{1+\alpha_{1} B}-\frac{\beta_{2} S I}{1+\alpha_{2} I}-\phi S-\mu_{1} S+\theta V \\
\phi S-\theta V-\mu_{1} V \\
\frac{\beta_{1} S B}{1+\alpha_{1} B}+\frac{\beta_{2} S I}{1+\alpha_{2} I}-\left(d+\alpha+\mu_{1}\right) I \\
\eta I-\mu_{2} B
\end{array}\right) .
$$

We have

$$
\begin{gathered}
\left.\frac{d S(t)}{d t}\right|_{S=0}=\mu_{1}+\theta V>0, \\
\left.\frac{d V(t)}{d t}\right|_{V=0}=\phi S>0, \\
\left.\frac{d I(t)}{d t}\right|_{I=0}=\frac{\beta_{1} S B}{1+\alpha_{1} B} \geq 0, \\
\left.\frac{d B(t)}{d t}\right|_{B=0}=\eta I \geq 0 .
\end{gathered}
$$

Therefore,

$$
\left.M_{i}\right|_{X_{i}(t)=0, X_{t} \in C_{+}^{4}} \geq 0, \quad i=1,2,3,4 .
$$

Due to Lemma 2 in [18], any solution of system (2) is such that $X(t) \in R_{+}^{4}$ for all $t \geq 0$. This completes the proof of Theorem 1 .

Theorem 2. All solutions $(S(t), V(t), I(t), B(t))$ of model (2) are bounded.

Proof. System (2) is split into two parts, the human population (i.e., $S(t), V(t)$, and $I(t))$ and pathogen population (i.e., $B(t)$ ). It follows from the first three equations of system (2) that

$$
\begin{aligned}
\frac{d(S+V+I)}{d t} & =\mu_{1}(1-S-I-V)-d I-\alpha I \\
& \leq \mu_{1}(1-S-I-V),
\end{aligned}
$$


then it follows that $\lim \sup _{t \rightarrow+\infty}(S+V+I) \leq 1$. From the first equation, we can get

$$
\begin{aligned}
\frac{d S(t)}{d t} & \leq \mu_{1}-\mu_{1} S-\phi S+\theta V \\
& \leq \mu_{1}+\theta-\left(\mu_{1}+\theta+\phi\right) S .
\end{aligned}
$$

Thus $d S(t) / d t \leq 0$, as $S(t) \geq\left(\mu_{1}+\theta\right) /\left(\mu_{1}+\theta+\phi\right)$. It is easy to obtain

$$
\begin{aligned}
\frac{d V(t)}{d t} & \leq \phi(1-V)-\left(\mu_{1}+\theta\right) V \\
& =\phi-\left(\mu_{1}+\theta+\phi\right) V .
\end{aligned}
$$

Thus $d V(t) / d t \leq 0$, as $V(t) \geq \phi /\left(\mu_{1}+\theta+\phi\right)$. From the last equation, we can obtain

$$
\frac{d B(t)}{d t} \leq \eta-\mu_{2} B
$$

Hence, $d B(t) / d t \leq 0$, when $B \geq \eta / \mu_{2}$. Therefore, all solutions $(S(t), V(t), I(t), B(t))$ of model (2) are bounded.

From above discussion, we can see that the feasible region of human population for system (2) is

$$
\begin{gathered}
\Omega_{H}=\left\{(S, V, I) \mid S+V+I \leq 1,0 \leq S \leq \frac{\mu_{1}+\theta}{\mu_{1}+\theta+\phi},\right. \\
\left.0 \leq V \leq \frac{\phi}{\mu_{1}+\theta+\phi}, I \geq 0\right\},
\end{gathered}
$$

and the feasible region of pathogen population for system (2) is

$$
\Omega_{B}=\left\{B \mid 0 \leq B \leq \frac{\eta}{\mu_{2}}\right\} .
$$

Define $\Omega=\Omega_{H} \times \Omega_{B}$. Let int $\Omega$ denote the interior of $\Omega$. It is easy to verify that the region $\Omega$ is a positively invariant region (i.e., the solutions with initial conditions in $\Omega$ remain in $\Omega$ ) with respect to system (2). Hence, we will consider the global stability of (2) in region $\Omega$.

\section{Equilibria}

In this section, we investigate the existence of equilibria of system (2). Solving the right hand side of model system (2) by equating it to zero, we obtain the following biologically relevant equilibria.

It is easy to see that model (2) always has a disease-free equilibrium (the absence of infection, i.e., $I=B=0$ ) $E_{0}\left(S_{0}, V_{0}, 0,0\right)$, where $S_{0}=\left(\mu_{1}+\theta\right) /\left(\mu_{1}+\theta+\phi\right)$ and $V_{0}=$ $\phi /\left(\mu_{1}+\theta+\phi\right)$.
In the following, we will discuss the existence and uniqueness of the endemic equilibrium. The components of the endemic equilibrium $E^{*}\left(S^{*}, V^{*}, I^{*}, B^{*}\right)$ satisfy

$$
\begin{gathered}
\mu_{1}-\frac{\beta_{1} S^{*} B^{*}}{1+\alpha_{1} B^{*}}-\frac{\beta_{2} S^{*} I^{*}}{1+\alpha_{2} I^{*}}-\phi S^{*}+\theta V^{*}-\mu_{1} S^{*}=0, \\
\phi S^{*}-\theta V^{*}-\mu_{1} V^{*}=0 \\
\frac{\beta_{1} S^{*} B^{*}}{1+\alpha_{1} B^{*}}+\frac{\beta_{2} S^{*} I^{*}}{1+\alpha_{2} I^{*}}-\left(d+\alpha+\mu_{1}\right) I^{*}=0 \\
\eta I^{*}-\mu_{2} B^{*}=0
\end{gathered}
$$

Substituting (13a), (13b), and (13d) into (13c), we obtain a single equation for $I^{*}$ :

$$
\begin{gathered}
\left(\frac{\beta_{1} \eta}{\mu_{2}+\alpha_{1} \eta I^{*}}+\frac{\beta_{2}}{1+\alpha_{2} I^{*}}\right) \frac{\left(\mu_{1}+\theta\right)\left[\mu_{1}-\left(d+\alpha+\mu_{1}\right) I^{*}\right]}{\mu_{1}\left(\mu_{1}+\theta+\phi\right)} I^{*} \\
-\left(d+\alpha+\mu_{1}\right) I^{*}=0 .
\end{gathered}
$$

After dropping the solution $I^{*}=0$, we obtain

$$
g_{1}\left(I^{*}\right)=g_{2}\left(I^{*}\right),
$$

where

$$
\begin{gathered}
g_{1}(I)=\frac{\left(\mu_{1}+\theta\right)\left[\mu_{1}-\left(d+\alpha+\mu_{1}\right) I\right]}{\mu_{1}\left(\mu_{1}+\theta+\phi\right)}, \\
g_{2}(I)=\frac{d+\alpha+\mu_{1}}{\left(\beta_{1} \eta /\left(\mu_{2}+\alpha_{1} \eta I\right)\right)+\left(\beta_{2} /\left(1+\alpha_{2} I\right)\right)} .
\end{gathered}
$$

Note that $g_{1}(I)$ represents a straight line with a negative slope and a vertical intercept $g_{1}(0)=\left(\mu_{1}+\theta\right) /\left(\mu_{1}+\theta+\phi\right)$. Meanwhile, we have

$$
\begin{aligned}
g_{2}^{\prime}(I)= & \frac{d+\alpha+\mu_{1}}{\left(\left(\beta_{1} \eta /\left(\mu_{2}+\alpha_{2} \eta I\right)\right)+\left(\mu_{2} /\left(1+\alpha_{2} I\right)\right)\right)^{2}} \\
& \times\left[\frac{\beta_{1} \alpha_{2} \eta^{2}}{\left(\mu_{2}+\alpha_{2} \eta I\right)^{2}}+\frac{\beta_{2} \alpha_{2}}{\left(1+\alpha_{2} I\right)^{2}}\right]>0 .
\end{aligned}
$$

We see that $g_{2}(I)$ is increasing for $I \geq 0$, and $g_{2}(0)=\left(\left(\mu_{1}+\right.\right.$ $\left.\theta) /\left(\mu_{1}+\theta+\phi\right)\right)\left(1 / \mathscr{R}_{v}\right)$, where

$$
\mathscr{R}_{v}=\frac{\left(\mu_{1}+\theta\right)\left(\beta_{2} \mu_{2}+\beta_{1} \eta\right)}{\mu_{2}\left(\mu_{1}+\theta+\phi\right)\left(d+\alpha+\mu_{1}\right)}
$$

is the control reproduction number of infection. When $R_{v}>1, g_{2}(0)<g_{1}(0)$. Hence, there is one and only one intersection between the curves of $g_{1}(I)$ and $g_{2}(I)$; that is, there is a unique solution $I^{*}$ to the equation $g_{1}\left(I^{*}\right)=g_{2}\left(I^{*}\right)$. Consequently, $S^{*}, V^{*}$, and $B^{*}$ are uniquely determined by $I^{*}$.

Theorem 3. System (2) has a unique endemic equilibrium when $\mathscr{R}_{v}>1$ and no positive endemic equilibrium when $\mathscr{R}_{v}<1$. 


\section{Stability of Disease-Free Equilibrium}

Now, we will discuss the local and global stability of the disease-free equilibrium.

Theorem 4. The disease-free equilibrium $E_{0}$ is locally asymptotically stable for $\mathscr{R}_{v}<1$ and unstable for $\mathscr{R}_{v}>1$.

Proof. The Jacobian matrix of system (2) at $X=E_{0}$ is

$$
\begin{aligned}
& J\left(E_{0}\right) \\
& =\left(\begin{array}{cccc}
-\mu_{1}-\phi & \theta & -\beta_{2} S_{0} & -\beta_{1} S_{0} \\
\phi & -\theta-\mu_{1} & 0 & 0 \\
0 & 0 & \beta_{2} S_{0}-\left(d+\alpha+\mu_{1}\right) & \beta_{1} S_{0} \\
0 & 0 & \eta & -\mu_{2}
\end{array}\right) .
\end{aligned}
$$

The characteristic polynomial of the matrix $J\left(E_{0}\right)$ is given by

$$
\operatorname{det}\left(\lambda I-J\left(E_{0}\right)\right)=a_{0} \lambda^{4}+a_{1} \lambda^{3}+a_{2} \lambda^{2}+a_{3} \lambda+a_{4},
$$

where

$$
\begin{gathered}
a_{0}=1, \\
a_{1}=2 \mu_{1}+\mu_{2}+\theta+\phi+\xi_{1}, \\
a_{2}=\left(\theta+2 \mu_{1}+\phi\right) \xi_{1}+\xi_{2}+\mu_{1}^{2} \\
+2 \mu_{1} \mu_{2}+(\theta+\phi)\left(\mu_{1}+\mu_{2}\right), \\
a_{3}=\left(2 \mu_{1}+\phi+\theta\right) \xi_{2}+\left(\mu_{1} \theta+\mu_{1}^{2}+\mu_{1} \phi\right) \xi_{1} \\
+\mu_{1} \mu_{2}\left(\mu_{1}+\theta+\phi\right), \\
a_{4}=\mu_{1} \mu_{2}\left(\theta+\phi+\mu_{1}\right)\left(d+\alpha+\mu_{1}\right)\left(1-\mathscr{R}_{v}\right), \\
a_{1} a_{2}-a_{3}=\xi_{1}\left(2 \mu_{1}+\phi\right)^{2}+4 \mu_{1}\left(\theta+\mu_{2}\right) \\
\left.+2 \mu_{2}(\phi+\theta)+\theta^{2}+2 \theta \phi\right) \\
+\xi_{2} \mu_{2}+\xi_{1}^{2}\left(\theta+2 \mu_{1}+\phi\right)+4 \mu_{1} \mu_{2}\left(\theta+\phi+\mu_{1}\right) \\
+\left(2 \mu_{1}+\theta\right) \mu_{2}^{2}+3 \mu_{1}^{2}(\phi+\theta) \\
+\left(\phi^{2}+\theta^{2}\right)\left(\mu_{1}+\mu_{2}\right)+2 \theta \phi \mu_{2}+2 \theta \phi \mu_{1}+2 \mu_{1}^{3} . \\
\xi_{1}=d+\alpha+\mu_{1}-\beta S_{0}, \\
\xi_{2}=\mu_{2}\left(d+\alpha+\mu_{1}\right)-\left(\beta_{2} S_{0} \mu_{2}+\beta_{1} S_{0} \eta\right) .
\end{gathered}
$$

If $\mathscr{R}_{v}<1$, then

$$
\mu_{2}\left(d+\alpha+\mu_{1}\right)>\beta_{2} S_{0} \mu_{2}+\beta_{1} S_{0} \eta \text {, i.e } \xi_{2}>0 .
$$

Further,

$$
d+\alpha+\mu_{1}>\beta_{2} S_{0} \text {, i.e } \xi_{1}>0 \text {. }
$$

So $a_{1}>0, a_{2}>0, a_{3}>0, a_{4}>0, a_{1} a_{2}-a_{3}>0$, and $a_{1} a_{2} a_{3}>a_{3}^{2}+a_{1}^{2} a_{4}$ (see Appendix A). Thus, using the RouthHurwitz criterion, all eigenvalues of $J\left(E_{0}\right)$ have negative real part; $E_{0}$ is local asymptotically stable for system (2). If $\mathscr{R}_{v} \geq$ 1 , then $a_{4} \leq 0$ and we show that $J\left(E_{0}\right)$ has at least one eigenvalue with nonnegative real parts. Consequently, $E_{0}$ is not asymptotically stable.

Theorem 5. When $\mathscr{R}_{v}<1$, the disease-free equilibrium is globally asymptotically stable.

We will prove the global stability of the disease-free equilibrium using Lemma 6.

Lemma 6 (see [19]). If a model system can be written in the form

$$
\frac{d \mathscr{X}}{d t}=P(\mathscr{X}, \mathscr{Z})
$$

$$
\frac{d \mathscr{Z}}{d t}=G(\mathscr{X}, \mathscr{Z}), \quad G(\mathscr{Z}, 0)=0 \text {, }
$$

where $\mathscr{X} \in R^{m}$ denotes (its components) the number of uninfected individuals and $\mathscr{Z} \in R^{n}$ denotes (its components) the number of infected individuals including latent and so forth, $U_{0}=\left(\mathscr{X}^{*}, 0\right)$ denotes the disease-free equilibrium of the system.

Assume that

(H1) for $d \mathscr{X} / d t=P(\mathscr{X}, 0), \mathscr{X}^{*}$ is globally asymptotically stable;

(H2) $G(\mathscr{X}, \mathscr{Z})=\mathscr{A} \mathscr{Z}-\widehat{G}(\mathscr{X}, \mathscr{Z}), \widehat{G}(\mathscr{X}, \mathscr{Z}) \geq 0$ for $(\mathscr{X}, \mathscr{Z}) \in \Omega$, where the Jacobian matrix $\mathscr{A}=$ $(\partial G / \partial \mathscr{Z})\left(\mathscr{X}^{*}, 0\right)$ is an Metzler matrix (the off-diagonal elements of $\mathscr{A}$ are nonnegative) and $\Omega$ is the region where the model makes biological sense. Then the fixed point $U_{0}=\left(\mathscr{X}^{*}, 0\right)$ is a globally asymptotically stable equilibrium of cholera model system (2) provided that $\mathscr{R}_{v}<1$.

We begin by showing condition (H1) as

$$
P(\mathscr{X}, 0)=\left(\begin{array}{c}
\mu_{1}-\mu_{1} S-\phi S+\theta V \\
\phi S-\theta V-\mu_{1} V
\end{array}\right) .
$$

For the equilibrium $U_{0}=\left(\mathscr{X}^{*}, 0\right)$, the system reduces to

$$
\begin{gathered}
\frac{d S(t)}{d t}=\mu_{1}-\phi S(t)-\mu_{1} S(t)+\theta V(t), \\
\frac{d V(t)}{d t}=\phi S(t)-\theta V(t)-\mu_{1} V(t) .
\end{gathered}
$$

The characteristic polynomial of the system is given by

$$
\left(\lambda+\mu_{1}\right)\left(\lambda+\theta+\phi+\mu_{1}\right)=0 .
$$

There are two negative characteristic foots: $\lambda_{1}=-\mu_{1}, \lambda_{2}=$ $-\theta-\phi-\mu_{1}$. Hence, $\mathscr{X}^{*}$ is always globally asymptotically stable. 

gives

Next, applying Lemma 6 to the cholera model system (2)

$$
\begin{aligned}
G( & \mathscr{X}, \mathscr{Z}) \\
= & \mathscr{A} \mathscr{Z}-\widehat{G}(\mathscr{X}, \mathscr{Z}) \\
= & \left(\begin{array}{cc}
\beta_{2} S_{0}-\left(d+\alpha+\mu_{1}\right) & \beta_{1} S_{0} \\
\eta & -\mu_{2}
\end{array}\right)\left(\begin{array}{l}
I \\
B
\end{array}\right) \\
& -\left(\begin{array}{cc}
\beta_{1} B\left(S_{0}-\frac{S}{1+\alpha_{1} B}\right)+\beta_{2} I \\
0
\end{array}\right.
\end{aligned}
$$

So $\mathscr{A}$ is a Metzler matrix. Meanwhile, we find $\widehat{G}(\mathscr{X}, \mathscr{Z}) \geq 0$. Hence, the disease-free equilibrium is globally asymptotically stable.

\section{Persistence}

Persistence is an important property of dynamical systems and of the systems in ecology, epidemics, and so forth, they are modeling. Biologically, persistence means the survival of all populations in future time. Mathematically, persistence of a system means that strictly positive solutions do not have any omega limit points on the boundary of the nonnegative cone [20]. In this section, we will present the persistence of system (2).

For various definitions of persistence [21, 22], we utilize the definitions of persistence developed by Freedman et al. [23]. System (2) can be defined to be uniformly persistent if

$$
\begin{gathered}
\min \left\{\liminf _{t \rightarrow \infty} S(t), \liminf _{t \rightarrow \infty} V(t), \liminf _{t \rightarrow \infty} I(t),\right. \\
\left.\liminf _{t \rightarrow \infty} B(t)\right\}>\epsilon
\end{gathered}
$$

for some $\epsilon>0$ for all initial points in int $\Omega$.

A uniform persistence result given in [23] requires the following hypothesis $(\mathrm{H})$ to be satisfied.

We denote that $E$ is a closed positively invariant subset of $X$ on which a continuous flow $\mathscr{F}$ is defined and $N$ is the maximal invariant set of $\partial \mathscr{F}$ on $\partial E$. Suppose $N$ is a closed invariant set and there exists a cover $\left\{N_{\alpha}\right\}_{\alpha \in A}$ of $N$, where $A$ is a nonempty index set; $N_{\alpha} \subset \partial E, N \subset \cup_{\alpha \in A} N_{\alpha}$, and $\left\{N_{\alpha}\right\}(\alpha \in$ $A)$ are pairwise disjoint closed invariant sets. Furthermore, we propose the following hypothesis.

Hypothesis $(\mathrm{H})$ :

(a) all $N_{\alpha}$ are isolated invariant sets of the flow $\mathscr{F}$;

(b) $\left\{N_{\alpha}\right\}_{\alpha \in A}$ is acyclic; that is, any finite subset of $\left\{N_{\alpha}\right\}_{\alpha \in A}$ does not form a cycle [24];

(c) any compact subset of $\partial E$ contains, at most, finitely many sets of $\left\{N_{\alpha}\right\}_{\alpha \in A}$.

Lemma 7 (see [24]). Let $E$ be a closed positively invariant subset of $X$ on which a continuous flow $\mathscr{F}$ is defined. Suppose there is a constant $\varepsilon>0$ such that $\mathscr{F}$ is point dissipative on
$\{X: x \in X, d(x, \partial E) \leq \varepsilon\} \cap$ int $E$ and the assumption $(H)$ holds. Then the flow $\mathscr{F}$ is uniformly persistent if and only if

$$
W^{+}\left(N_{\alpha}\right) \cap\{X: x \in X, d(x, \partial E) \leq \varepsilon\} \cap \operatorname{int} E=\varnothing
$$

for any $\alpha \in A$, where $W^{+}\left(N_{\alpha}\right)=\left\{y \in X: \Lambda^{+}(y) \subset N_{\alpha}\right\}$.

Now, we can obtain the following result.

Theorem 8. System (2) is uniformly persistent in int $\Omega$ if $\mathscr{R}_{v}>$ 1.

Proof. Suppose $\mathscr{R}_{v}>1$. We show that system (2) satisfies all the conditions of Lemma 7. Choose $X=\mathbb{R}^{4}$ and $E=\Omega$. The vector field of system (2) is transversal to the boundary of $\Omega$ on its faces except the $S$-axis and $V$-axis, which are invariant with respect to system (2) and on the $S$-axis and $V$ axis the equations for $S$ and $V$ are $d S(t) / d t=\mu_{1}-\phi S(t)-$ $\mu_{1} S(t)+\theta V(t)$, and $d V(t) / d t=\phi S(t)-\theta V(t)-\mu_{1} V(t)$, which implies that $S(t) \rightarrow\left(\mu_{1}+\theta\right) /\left(\mu_{1}+\theta+\phi\right)$ and $V(t) \rightarrow$ $\phi /\left(\mu_{1}+\theta+\phi\right)$ as $t \rightarrow \infty$. Therefore, $E_{0}$ is the only $\omega$ limit point on the boundary of $\Omega$. As the maximal invariant set on the boundary $\partial \Omega$ of $\Omega$ is the singleton $\left\{E_{0}\right\}$ and $E_{0}$ is isolated when $\mathscr{R}_{v}<1$, thus the hypothesis $(\mathrm{H})$ holds for system (2). The flow induced by $f(x)$ is point dissipative $\partial E$ by the positive invariance of $E$. Because of $W^{+}(N)=\{y \in X$ : $\left.\Lambda^{+}(y) \subset N\right\}$, where $\Lambda^{+}(y)$ is the omega limit set of $y$, when $\mathscr{R}_{v}<1$, we have that $E$ is contained in the set $W^{+}(N)$ and for $\mathscr{R}_{v}>1, W^{+}(N)=\varnothing$. Therefore, the uniform persistence of system (2) is equivalent to $P_{0}$ being unstable, and the theorem is proved.

Remark 9. Theorems 3 and 8 show that $\mathscr{R}_{v}$ is a threshold parameter for the model; that is, when $\mathscr{R}_{v}<1$, its epidemiological implication is that the infected fraction of the population vanishes, so the cholera dies out; when $\mathscr{R}_{v}>$ 1 , the disease is endemic and the infected fraction remains above a certain positive level for sufficiently large time.

\section{Stability of the Endemic Equilibrium}

6.1. Local Stability of the Endemic Equilibrium. Now we consider the case with $\mathscr{R}_{v}>1$. The stability of the endemic equilibrium is established as follows.

Theorem 10. If $\mathscr{R}_{v}>1, E^{*}\left(S^{*}, V^{*}, I^{*}, B^{*}\right)$ is locally asymptotically stable.

Proof. Let

$$
\begin{gathered}
J_{1}=\frac{\beta_{1} B^{*}}{1+\alpha_{1} B^{*}}+\frac{\beta_{2} I^{*}}{1+\alpha_{2} I^{*}}, \\
J_{2}=\frac{\beta_{2} S^{*}}{\left(1+\alpha_{2} I^{*}\right)^{2}}, \\
J_{3}=\frac{\beta_{1} S^{*}}{\left(1+\alpha_{1} B^{*}\right)^{2}} .
\end{gathered}
$$


The Jacobian matrix at $E^{*}\left(S^{*}, V^{*}, I^{*}, B^{*}\right)$ is

$$
\begin{aligned}
& J\left(E^{*}\right) \\
& \quad=\left(\begin{array}{cccc}
-J_{1}-\mu_{1}-\phi & \theta & -J_{2} & -J_{3} \\
\phi & -\theta-\mu_{1} & 0 & 0 \\
J_{1} & 0 & J_{2}-\left(d+\alpha+\mu_{1}\right) & J_{3} \\
0 & 0 & \eta & -\mu_{2}
\end{array}\right) .
\end{aligned}
$$

The characteristic polynomial of the matrix $J\left(E^{*}\right)$ is given by

$$
\operatorname{det}\left(\lambda I-J\left(E^{*}\right)\right)=b_{0} \lambda^{4}+b_{1} \lambda^{3}+b_{2} \lambda^{2}+b_{3} \lambda+b_{4}
$$

where

$$
\begin{gathered}
b_{0}=1 \\
b_{1}=J_{1}+2 \mu_{1}+\mu_{2}+\theta+\phi+\omega_{1}, \\
b_{2}=\left(\theta+2 \mu_{1}+\phi\right) \omega_{1}+\omega_{2}+\mu_{1}^{2}+2 \mu_{1} \mu_{2} \\
+(\theta+\phi)\left(\mu_{1}+\mu_{2}\right)+\left(\alpha+2 \mu_{1}+\mu_{2}+d\right) J_{1}, \\
b_{3}=\left(\theta+\mu_{1}+\phi\right) \mu_{1} \omega_{1}+\left(2 \mu_{1}+\theta+\phi\right) \omega_{2} \\
+\mu_{1} \mu_{2}\left(\theta+\mu_{1}+\phi\right)+\alpha J_{1}\left(\theta+\mu_{1}+\mu_{2}\right) \\
+J_{1}\left(\mu_{1}^{2}+(d+\theta)\left(\mu_{1}+\mu_{2}\right)+2 \mu_{1} \mu_{2}+\theta d\right), \\
b_{4}=\left(\phi+\theta+\mu_{1}\right) \mu_{1} \omega_{2}+\left(d+\alpha+\theta+\mu_{1}\right) \mu_{1} \mu_{2} J_{1} \\
+(d+\alpha) \theta \mu_{2} J_{1}, \\
b_{1} b_{2}-b_{3}=\omega_{1}\left\{4 \mu_{1}\left(\theta+\phi+\mu_{1}+\mu_{2}\right)+(\phi+\theta)^{2}\right. \\
+J_{1}\left(\alpha+2 \theta+\mu_{2}+d+\phi\right) J_{1}+2 \theta \mu_{2} \\
\left.+J_{1} \cdot \phi+2 \mu_{2} \phi\right\}
\end{gathered}
$$

$$
\begin{aligned}
& +\omega_{1}^{2}\left(\theta+2 \mu_{1}+\phi\right)+\omega_{1} \omega_{2}+\omega_{2}\left(\mu_{2}+J_{1}\right) \\
& +\mu_{2}^{2}\left(2 \mu_{1}+\theta+J_{1}+\phi\right) \\
& +J_{1}^{2}\left(\alpha+2 \mu_{1}+\mu_{2}+d+\theta\right)+3 \mu_{1}^{2}(\phi+\theta) \\
& +(\theta+\phi)^{2}\left(\mu_{1}+\mu_{2}\right)+\theta^{2} J_{1} \\
& +\phi J_{1}\left(\theta+\alpha+d+3 \mu_{1}\right) \\
& +4 \mu_{1} \mu_{2}\left(J_{1}+\theta+\phi\right)+2 J_{1} \mu_{2}(\theta+\phi) \\
& +4 \mu_{1}^{2}\left(J_{1}+\mu_{2}\right)+2 \mu_{1}^{3}+J_{1} \mu_{1}(d+\alpha+4 \theta) . \\
& \quad \omega_{1}=d+\alpha+\mu_{1}-J_{2}, \\
& \quad \omega_{2}=\mu_{2}\left(d+\alpha+\mu_{1}\right)-\left(J_{2} \mu_{2}+J_{3} \eta\right) .
\end{aligned}
$$

Based on (13c) and (13d), we have $\mu_{2}\left(d+\alpha+\mu_{1}\right)=\left(\beta_{1} \eta S^{*} /(1+\right.$ $\left.\left.\alpha_{1} B^{*}\right)\right)+\left(\beta_{2} \mu_{2} S^{*} /\left(1+\alpha_{2} I^{*}\right)\right)$. It is then easy to observe that

$$
\mu_{2}\left(d+\alpha+\mu_{1}\right)>\mu_{2} J_{2}+\eta J_{3} \text {, i.e } \omega_{2}>0 \text {. }
$$

Further,

$$
d+\alpha+\mu_{1}>J_{2} \text {, i.e } \omega_{1}>0 \text {. }
$$

So $b_{1}>0, b_{2}>0, b_{3}>0, b_{4}>0, b_{1} b_{2}-b_{3}>0$, and $b_{1} b_{2} b_{3}>b_{3}^{2}+$ $b_{1}^{2} b_{4}$ (see Appendix B). Using the well-known Routh-Hurwitz criterion, the proof is thus complete.

\subsection{Global Stability of the Endemic Equilibrium}

Theorem 11. When $\mathscr{R}_{v}>1, E^{*}\left(S^{*}, V^{*}, I^{*}, B^{*}\right)$ is globally asymptotically stable in $\Omega \backslash \Omega_{0}$ if $d+\alpha \leq \mu_{1}+\theta$ and $\left(2 \beta_{2}\left(\mu_{1}+\theta\right) /\left(\mu_{1}+\theta+\phi\right)\right)+\theta \leq \mu_{1}$.

This approach to global dynamics is developed in the papers of Smith [25] and Li and Muldowney [26-28]. Let $f=\left(f_{1}, f_{2}, f_{3}, f_{4}\right)^{T}$, where $f_{1}, f_{2}, f_{3}$, and $f_{4}$ represent the right-hand sides of system (2), respectively. Furthermore, let $x=(S, V, E, I)^{T}$. Then, the Jacobian matrix for system (2) is

$$
J=\left(\begin{array}{cccc}
-\left(\frac{\beta_{1} B}{1+\alpha_{1} B}+\frac{\beta_{2} I}{1+\alpha_{2} I}\right)-\mu_{1}-\phi & \theta & -\frac{\beta_{2} S}{\left(1+\alpha_{2} I\right)^{2}} & -\frac{\beta_{1} S}{\left(1+\alpha_{1} B\right)^{2}} \\
\phi & -\theta-\mu_{1} & 0 & 0 \\
\frac{\beta_{1} B}{1+\alpha_{1} B}+\frac{\beta_{2} I}{1+\alpha_{2} I} & 0 & \frac{\beta_{2} S}{\left(1+\alpha_{2} I\right)^{2}}-\left(d+\alpha+\mu_{1}\right) & \frac{\beta_{1} S}{\left(1+\alpha_{1} B\right)^{2}} \\
0 & 0 & \eta & -\mu_{2}
\end{array}\right) .
$$

The second additive compound matrix (see Appendix C for details) of $J$ is 
$J^{[2]}=\left(\begin{array}{cccccc}j_{11} & 0 & 0 & \frac{\beta_{2} S}{\left(1+\alpha_{2} I\right)^{2}} & \frac{\beta_{1} S}{\left(1+\alpha_{1} B\right)^{2}} & 0 \\ 0 & j_{22} & \frac{\beta_{1} S}{\left(1+\alpha_{1} B\right)^{2}} & \theta & 0 & \frac{\beta_{1} S}{\left(1+\alpha_{1} B\right)^{2}} \\ 0 & \eta & j_{33} & 0 & \theta & -\frac{\beta_{2} S}{\left(1+\alpha_{2} I\right)^{2}} \\ -\left(\frac{\beta_{1} B}{1+\alpha_{1} B}+\frac{\beta_{2} I}{1+\alpha_{2} I}\right) & \phi & 0 & j_{44} & \frac{\beta_{1} S}{\left(1+\alpha_{1} B\right)^{2}} & 0 \\ 0 & 0 & \phi & \eta & j_{55} & 0 \\ 0 & 0 & \frac{\beta_{1} B}{1+\alpha_{1} B}+\frac{\beta_{2} I}{1+\alpha_{2} I} & 0 & 0 & j_{66}\end{array}\right)$,

where

$$
\begin{aligned}
& j_{11}=j_{33}+\mu_{2}-\mu_{1}-\theta, \\
& j_{22}=j_{44}+\theta-\frac{\beta_{1} B}{1+\alpha_{1} B}-\frac{\beta_{2} I}{1+\alpha_{2} I}-\phi, \\
& j_{33}=-\frac{\beta_{1} B}{1+\alpha_{1} B}-\frac{\beta_{2} I}{1+\alpha_{2} I}-\mu_{1}-\phi-\mu_{2}, \\
& j_{44}=\frac{\beta_{2} S}{\left(1+\alpha_{2} I\right)^{2}}-2 \mu_{1}-\theta-d-\alpha, \\
& j_{55}=-\mu_{1}-\mu_{2}-\theta, \\
& j_{66}=\frac{\beta_{2} S}{\left(1+\alpha_{2} I\right)^{2}}-\mu_{1}-\mu_{2}-d-\alpha .
\end{aligned}
$$

$$
Q=\left(\begin{array}{cccccc}
\frac{1}{I} & 0 & 0 & 0 & 0 & 0 \\
0 & \frac{1}{I} & 0 & 0 & 0 & 0 \\
0 & 0 & 0 & \frac{1}{I} & 0 & 0 \\
0 & 0 & \frac{1}{B} & 0 & 0 & 0 \\
0 & 0 & 0 & 0 & \frac{1}{B} & 0 \\
0 & 0 & 0 & 0 & 0 & \frac{1}{B}
\end{array}\right)
$$

Then we have

$$
\begin{aligned}
\mathfrak{B} & =Q_{f} Q^{-1}+Q \frac{\partial f^{[2]}}{\partial x} Q^{-1} \\
& =\left(\begin{array}{cccccc}
g_{11} & 0 & \frac{\beta_{2} S}{\left(1+\alpha_{2} I\right)^{2}} & 0 & \frac{\beta_{1} B S}{I\left(1+\alpha_{1} B\right)^{2}} & 0 \\
0 & g_{22} & \theta & \frac{\beta_{1} B S}{I\left(1+\alpha_{1} B\right)^{2}} & 0 & \frac{\beta_{1} B S}{I\left(1+\alpha_{1} B\right)^{2}} \\
-\frac{\beta_{1} B}{1+\alpha_{1} B}-\frac{\beta_{2} I}{1+\alpha_{2} I} & \phi & g_{33} & 0 & \frac{\beta_{1} B S}{I\left(1+\alpha_{1} B\right)^{2}} & 0 \\
0 & \frac{\eta I}{B} & 0 & g_{44} & \theta & -\frac{\beta_{2} S}{\left(1+\alpha_{2} I\right)^{2}} \\
0 & 0 & \frac{\eta I}{B} & \phi & g_{55} & 0 \\
0 & 0 & 0 & \frac{\beta_{1} B}{1+\alpha_{1} B}+\frac{\beta_{2} I}{1+\alpha_{2} I} & 0 & g_{66}
\end{array}\right),
\end{aligned}
$$

where

$$
\begin{array}{ll}
g_{11}=-\frac{\beta_{1} B S}{I\left(1+\alpha_{1} B\right)}-\frac{\beta_{2} S}{1+\alpha_{2} I}-\theta+d+\alpha+\frac{\eta I}{B}+g_{44}, & g_{33}=-\frac{\beta_{1} B S}{I\left(1+\alpha_{1} B\right)}-\frac{\beta_{2} \alpha_{2} S I}{\left(1+\alpha_{2} I\right)^{2}}-\mu_{1}-\theta, \\
g_{22}=-\frac{\beta_{1} B S}{I\left(1+\alpha_{1} B\right)}-\frac{\beta_{2} \alpha_{2} S I}{\left(1+\alpha_{2} I\right)^{2}}+\frac{\eta I}{B}+g_{44}, & g_{44}=-\frac{\beta_{1} B}{1+\alpha_{1} B}-\frac{\beta_{2} I}{1+\alpha_{2} I}-\frac{\eta I}{B}-\mu_{1}-\phi,
\end{array}
$$




$$
\begin{aligned}
& g_{55}=-\frac{\eta I}{B}-\mu_{1}-\theta, \\
& g_{66}=\frac{\beta_{2} S}{\left(1+\alpha_{2} I\right)^{2}}-\frac{\eta I}{B}-\mu_{1}-d-\alpha .
\end{aligned}
$$

In (41), $Q_{f}$ is the directional derivative of $Q$ in the direction of the vector field $f$ in system (2).

Here, we will use the theorem in [28] to give a sufficient condition on the parameters, which when satisfied, implies that the endemic equilibrium is globally asymptotically stable.

Lemma 12 (see [28]). If $\Omega_{1}$ is a compact absorbing subset in the interior of $\Omega$ and there exists $v>0$ and a Lozinskii measure $\bar{\mu}(\mathfrak{B}) \leq-v$ for all $x \in \Omega_{1}$, then every omega point of system (2) in int $\Omega$ is an equilibrium in $\Omega$.

For $R_{v}>1$, the disease-free equilibrium is repelling towards the interior. In fact, there is a compact absorbing set in int $\Omega$ which attracts all orbits that intersect int $\Omega$. This gives the following results.

Corollary 13. If $R_{v}>1$ and there exists a Lozinskir measure $\bar{\mu}(\mathfrak{B})$ such that $\bar{\mu}(\mathfrak{B})<0$ for all $x \in$ int $\Omega$, then each orbit of system (2) which intersects int $\Omega$ limits to the endemic equilibrium.

For a norm $\|\cdot\|$ on $\mathbb{R}^{n}$, the Lozinskiǔ measure $\bar{\mu}$ associated with $\|\cdot\|$ can be evaluated for an $n \times n$ matrix $\mathscr{T}$ as

$$
\begin{aligned}
& \bar{\mu}(\mathscr{T}) \\
& =\inf \left\{\sigma: D_{+}\|z\| \leq \sigma\|z\|, \text { for all solutions of } z^{\prime}=\mathscr{T} z\right\},
\end{aligned}
$$

where $D_{+}$is the right-hand derivative [29]. Hence, if we can find a norm on $\mathbb{R}^{6}$ for which the associated Lozinskii measure satisfied $\bar{\mu}(\mathscr{B})<0$ for all $x \in$ int $\Omega$ then the endemic equilibrium is globally asymptotically stable for $\mathscr{R}_{v}>1$.

We now define a norm on $\mathbb{R}^{6}$ [30] for which the definition varies from one orthant to another. Let

$$
\|z\|=\max \left\{U_{1}, U_{2}\right\},
$$

where $z \in \mathbb{R}^{6}$, with components $z_{i}(i=1,2,3,4,5,6)$ and

$$
U_{1}\left(z_{1}, z_{2}, z_{3}\right)=\left\{\begin{array}{l}
\max \left\{\left|z_{1}\right|,\left|z_{2}\right|+\left|z_{3}\right|\right\}, \\
\quad \text { if } \operatorname{sgn}\left(z_{1}\right)=\operatorname{sgn}\left(z_{2}\right)=\operatorname{sgn}\left(z_{3}\right), \\
\max \left\{\left|z_{2}\right|,\left|z_{1}\right|+\left|z_{3}\right|\right\}, \\
\quad \text { if } \operatorname{sgn}\left(z_{1}\right)=\operatorname{sgn}\left(z_{2}\right)=-\operatorname{sgn}\left(z_{3}\right), \\
\max \left\{\left|z_{1}\right|,\left|z_{2}\right|,\left|z_{3}\right|\right\}, \\
\quad \text { if } \operatorname{sgn}\left(z_{1}\right)=-\operatorname{sgn}\left(z_{2}\right)=\operatorname{sgn}\left(z_{3}\right), \\
\max \left\{\left|z_{1}\right|+\left|z_{3}\right|,\left|z_{2}\right|+\left|z_{3}\right|\right\}, \\
\quad \text { if }-\operatorname{sgn}\left(z_{1}\right)=\operatorname{sgn}\left(z_{2}\right)=\operatorname{sgn}\left(z_{3}\right),
\end{array}\right.
$$

$$
U_{2}\left(z_{4}, z_{5}, z_{6}\right)=\left\{\begin{array}{l}
\left|z_{4}\right|+\left|z_{5}\right|+\left|z_{6}\right|, \\
\quad \text { if } \operatorname{sgn}\left(z_{4}\right)=\operatorname{sgn}\left(z_{5}\right)=\operatorname{sgn}\left(z_{6}\right), \\
\max \left\{\left|z_{4}\right|+\left|z_{5}\right|,\left|z_{4}\right|+\left|z_{6}\right|\right\}, \\
\quad \text { if } \operatorname{sgn}\left(z_{4}\right)=\operatorname{sgn}\left(z_{5}\right)=-\operatorname{sgn}\left(z_{6}\right), \\
\max \left\{\left|z_{5}\right|,\left|z_{4}\right|+\left|z_{6}\right|\right\}, \\
\quad \text { if } \operatorname{sgn}\left(z_{4}\right)=-\operatorname{sgn}\left(z_{5}\right)=\operatorname{sgn}\left(z_{6}\right), \\
\max \left\{\left|z_{4}\right|+\left|z_{6}\right|,\left|z_{5}\right|+\left|z_{6}\right|\right\}, \\
\quad \text { if }-\operatorname{sgn}\left(z_{4}\right)=\operatorname{sgn}\left(z_{5}\right)=\operatorname{sgn}\left(z_{6}\right) .
\end{array}\right.
$$

Theorem 14. Assume that

$$
\begin{gathered}
d+\alpha \leq \mu_{1}+\theta, \\
\frac{2 \beta_{2}\left(\mu_{1}+\theta\right)}{\left(\mu_{1}+\theta+\phi\right)}+\theta \leq \mu_{1} .
\end{gathered}
$$

Then there exits $\sigma=\max \left\{-\mu_{1}, d+\alpha-\mu_{1}-\theta,\left(2 \beta_{2}\left(\mu_{1}+\theta\right) /\left(\mu_{1}+\right.\right.\right.$ $\left.\theta+\phi))+\theta-\mu_{1}\right\}<0$, such that $D_{+}\|z\| \leq \sigma\|z\|$ for all $z \in \mathbb{R}^{6}$.

Proof. We should show that

$$
\begin{aligned}
& D_{+}\|z\| \\
& \leq \max \left\{-\mu_{1}, d+\alpha-\mu_{1}-\theta, \frac{2 \beta_{2}\left(\mu_{1}+\theta\right)}{\left(\mu_{1}+\theta+\phi\right)}+\theta-\mu_{1}\right\}\|z\| .
\end{aligned}
$$

Case $1\left(U_{1}(z)>U_{2}(z)\right.$ and $\left.z_{1}, z_{2}, z_{3}>0\right)$. In this case, $\|z\|=$ $\max \left\{\left|z_{1}\right|,\left|z_{2}\right|+\left|z_{3}\right|\right\}$.

Subcase $1.1\left(\left|z_{1}\right|>\left|z_{2}\right|+\left|z_{3}\right|\right)$. Then $\|z\|=\left|z_{1}\right|=z_{1}$ and $U_{2}(z)<\left|z_{1}\right|$. Taking the right-hand derivative of $\|z\|$, we obtain

$$
\begin{aligned}
D_{+}\|z\|= & z_{1}^{\prime} \\
= & \left(-\frac{\beta_{1} B S}{I\left(1+\alpha_{1} B\right)}-\frac{\beta_{2} S}{1+\alpha_{2} I}-\frac{\beta_{1} B}{1+\alpha_{1} B}-\frac{\beta_{2} I}{1+\alpha_{2} I}\right. \\
& \left.+d+\alpha-\mu_{1}-\theta-\phi\right) z_{1} \\
& +\frac{\beta_{2} S}{\left(1+\alpha_{2} I\right)^{2}} z_{3}+\frac{\beta_{1} B S}{I\left(1+\alpha_{1} B\right)^{2}} z_{5} .
\end{aligned}
$$

Since $\left|z_{5}\right| \leq U_{2}(z)<\left|z_{1}\right|,\left|z_{3}\right| \leq\left|z_{1}\right|$, we have

$$
\begin{aligned}
D_{+}\|z\| \leq & -\frac{\beta_{1} B S}{I\left(1+\alpha_{1} B\right)}-\frac{\beta_{2} S}{1+\alpha_{2} I}-\frac{\beta_{1} B}{1+\alpha_{1} B}-\frac{\beta_{2} I}{1+\alpha_{2} I} \\
& \left.+d+\alpha-\mu_{1}-\theta-\phi\right)\left|z_{1}\right| \\
& +\frac{\beta_{2} S}{\left(1+\alpha_{2} I\right)^{2}}\left|z_{1}\right|+\frac{\beta_{1} B S}{I\left(1+\alpha_{1} B\right)^{2}}\left|z_{1}\right|
\end{aligned}
$$




$$
\begin{aligned}
= & -\frac{\beta_{1} \alpha_{1} B^{2} S}{I\left(1+\alpha_{1} B\right)^{2}}-\frac{\beta_{2} \alpha_{2} S I}{\left(1+\alpha_{2} I\right)^{2}}-\frac{\beta_{1} B}{1+\alpha_{1} B} \\
& \left.-\frac{\beta_{2} I}{1+\alpha_{2} I}+d+\alpha-\mu_{1}-\theta-\phi\right)\left|z_{1}\right| .
\end{aligned}
$$

Hence,

$$
\begin{aligned}
D_{+}\|z\| \leq & -\frac{\beta_{1} \alpha_{1} B^{2} S}{I\left(1+\alpha_{1} B\right)^{2}}-\frac{\beta_{2} \alpha_{2} S I}{\left(1+\alpha_{2} I\right)^{2}}-\frac{\beta_{1} B}{1+\alpha_{1} B} \\
& \left.-\frac{\beta_{2} I}{1+\alpha_{2} I}+d+\alpha-\mu_{1}-\theta-\phi\right)\|z\| .
\end{aligned}
$$

It is easy to see that (50) also holds for $U_{1}>U_{2}$ and $z_{1}, z_{2}, z_{3}<0$ when $\left|z_{1}\right|>\left|z_{2}\right|+\left|z_{3}\right|$, which can be obtained by linearity.

Subcase $1.2\left(\left|z_{1}\right|<\left|z_{2}\right|+\left|z_{3}\right|\right)$. Then $\|z\|=\left|z_{2}\right|+\left|z_{3}\right|=z_{2}+z_{3}$ and $U_{2}(z)<\left|z_{2}\right|+\left|z_{3}\right|$.

Since $\left|z_{4}+z_{5}+z_{6}\right| \leq U_{2}(z)<\left|z_{2}\right|+\left|z_{3}\right|$, taking the righthand derivative of $\|z\|$, we obtain

$$
\begin{aligned}
& D_{+}\|z\|=z_{2}^{\prime}+z_{3}^{\prime} \\
& =\left(-\frac{\beta_{1} B}{1+\alpha_{1} B}-\frac{\beta_{2} I}{1+\alpha_{2} I}\right) z_{1} \\
& +\left(-\frac{\beta_{1} B S}{I\left(1+\alpha_{1} B\right)}-\frac{\beta_{2} \alpha_{2} S I}{\left(1+\alpha_{2} I\right)^{2}}\right. \\
& \left.-\frac{\beta_{1} B}{1+\alpha_{1} B}-\frac{\beta_{2} I}{1+\alpha_{2} I}-\mu_{1}\right) z_{2} \\
& +\left(-\frac{\beta_{1} B S}{I\left(1+\alpha_{1} B\right)}-\frac{\beta_{2} \alpha_{2} S I}{\left(1+\alpha_{2} I\right)^{2}}-\mu_{1}\right) z_{3} \\
& +\frac{\beta_{1} B S}{I\left(1+\alpha_{1} B\right)^{2}}\left(z_{4}+z_{5}+z_{6}\right) \\
& \leq\left(-\frac{\beta_{1} B S}{I\left(1+\alpha_{1} B\right)}-\frac{\beta_{2} \alpha_{2} S I}{\left(1+\alpha_{2} I\right)^{2}}-\mu_{1}\right)\left(\left|z_{2}\right|+\left|z_{3}\right|\right) \\
& +\frac{\beta_{1} B S}{I\left(1+\alpha_{1} B\right)^{2}}\left(\left|z_{4}+z_{5}+z_{6}\right|\right) \\
& \leq\left(-\frac{\beta_{1} \alpha_{1} B^{2} S}{I\left(1+\alpha_{1} B\right)^{2}}-\frac{\beta_{2} \alpha_{2} S I}{\left(1+\alpha_{2} I\right)^{2}}-\mu_{1}\right)\left(\left|z_{2}\right|+\left|z_{3}\right|\right) .
\end{aligned}
$$

Therefore,

$$
D_{+}\|z\| \leq\left(-\frac{\beta_{1} \alpha_{1} B^{2} S}{I\left(1+\alpha_{1} B\right)^{2}}-\frac{\beta_{2} \alpha_{2} S I}{\left(1+\alpha_{2} I\right)^{2}}-\mu_{1}\right)\|z\| .
$$

It is easy to see that (52) also holds for $U_{1}>U_{2}$ and $z_{1}, z_{2}, z_{3}<$ 0 when $\left|z_{1}\right|<\left|z_{2}\right|+\left|z_{3}\right|$, which can be obtained by linearity.

Case $2\left(U_{1}(z)>U_{2}(z)\right.$ and $\left.z_{1}<0<z_{2}, z_{3}\right)$. In this case, $\|z\|=\max \left\{\left|z_{1}\right|+\left|z_{3}\right|,\left|z_{2}\right|+\left|z_{3}\right|\right\}$.

Subcase 2.1 $\left(\left|z_{1}\right|>\left|z_{2}\right|\right)$. Then $\|z\|=\left|z_{1}\right|+\left|z_{3}\right|=-z_{1}+z_{3}$ and $U_{2}(z)<\left|z_{1}\right|+\left|z_{3}\right|$. Taking the right-hand derivative of $\|z\|$, we obtain

$$
\begin{aligned}
D_{+}\|z\|= & -z_{1}^{\prime}+z_{3}^{\prime} \\
= & \left(\frac{\beta_{1} B S}{I\left(1+\alpha_{1} B\right)}+\frac{\beta_{2} S}{1+\alpha_{2} I}-d-\alpha+\mu_{1}+\theta+\phi\right) z_{1} \\
& +\phi z_{2}+\left(-\frac{\beta_{2} S}{1+\alpha_{2} I}-\frac{\beta_{1} B S}{I\left(1+\alpha_{1} B\right)}-\mu_{1}-\theta\right) z_{3} \\
\leq & \left(-\frac{\beta_{1} B S}{I\left(1+\alpha_{1} B\right)}-\frac{\beta_{2} S}{1+\alpha_{2} I}+d+\alpha-\mu_{1}-\theta\right)\left|z_{1}\right| \\
& +\left(-\frac{\beta_{2} S}{1+\alpha_{2} I}-\frac{\beta_{1} B S}{I\left(1+\alpha_{1} B\right)}-\mu_{1}-\theta\right)\left|z_{3}\right| \\
\leq & \left(-\frac{\beta_{1} B S}{I\left(1+\alpha_{1} B\right)}-\frac{\beta_{2} S}{1+\alpha_{2} I}+d+\alpha-\mu_{1}-\theta\right) \\
& \times\left(\left|z_{1}\right|+\left|z_{3}\right|\right) .
\end{aligned}
$$

Therefore,

$$
D_{+}\|z\| \leq\left(-\frac{\beta_{1} B S}{I\left(1+\alpha_{1} B\right)}-\frac{\beta_{2} S}{1+\alpha_{2} I}+d+\alpha-\mu_{1}-\theta\right)\|z\| .
$$

It is easy to see that (54) also holds for $U_{1}>U_{2}$ and $z_{2}, z_{3}<$ $0<z_{1}$ when $\left|z_{1}\right|>\left|z_{2}\right|$, which can be obtained by linearity.

Subcase $2.2\left(\left|z_{1}\right|<\left|z_{2}\right|\right)$. Then $\|z\|=\left|z_{2}\right|+\left|z_{3}\right|=z_{2}+z_{3}$ and $U_{2}(z)<\left|z_{2}\right|+\left|z_{3}\right|$. Taking the right-hand derivative of $\|z\|$, since $\left|z_{4}+z_{5}+z_{6}\right| \leq U_{2}(z)<\left|z_{2}\right|+\left|z_{3}\right|$, we obtain

$$
\begin{aligned}
D_{+}\|z\|= & z_{2}^{\prime}+z_{3}^{\prime} \\
= & \left(-\frac{\beta_{1} B}{1+\alpha_{1} B}-\frac{\beta_{2} I}{1+\alpha_{2} I}\right) z_{1} \\
& +\left(-\frac{\beta_{1} B S}{I\left(1+\alpha_{1} B\right)}-\frac{\beta_{2} \alpha_{2} S I}{\left(1+\alpha_{2} I\right)^{2}}-\frac{\beta_{1} B}{1+\alpha_{1} B}\right. \\
& \left.\quad-\frac{\beta_{2} I}{1+\alpha_{2} I}-\mu_{1}\right) z_{2}
\end{aligned}
$$




$$
\begin{aligned}
& +\left(-\frac{\beta_{1} B S}{I\left(1+\alpha_{1} B\right)}-\frac{\beta_{2} \alpha_{2} S I}{\left(1+\alpha_{2} I\right)^{2}}-\mu_{1}\right) z_{3} \\
& +\frac{\beta_{1} B S}{I\left(1+\alpha_{1} B\right)^{2}}\left(z_{4}+z_{5}+z_{6}\right) \\
& \leq\left(\frac{\beta_{1} B}{1+\alpha_{1} B}+\frac{\beta_{2} I}{1+\alpha_{2} I}\right)\left|z_{1}\right| \\
& +\left(-\frac{\beta_{1} B S}{I\left(1+\alpha_{1} B\right)}-\frac{\beta_{2} \alpha_{2} S I}{\left(1+\alpha_{2} I\right)^{2}}-\frac{\beta_{1} B}{1+\alpha_{1} B}\right. \\
& +\left(-\frac{\beta_{1} B S}{I\left(1+\alpha_{1} B\right)}-\frac{\beta_{2} \alpha_{2} S I}{\left(1+\alpha_{2} I\right)^{2}}-\mu_{1}\right) z_{3} \\
& +\frac{\beta_{1} B S}{I\left(1+\alpha_{1} B\right)^{2}}\left(\left|z_{4}+z_{5}+z_{6}\right|\right) \\
& \leq\left(-\frac{\beta_{1} \alpha_{1} B^{2} S}{I\left(1+\alpha_{1} B\right)}-\frac{\beta_{2} \alpha_{2} S I}{\left(1+\alpha_{2} I\right)^{2}}-\mu_{1}\right)\left(\left|z_{2}\right|+\left|z_{3}\right|\right)
\end{aligned}
$$

Therefore,

$$
D_{+}\|z\| \leq\left(-\frac{\beta_{1} \alpha_{1} B^{2} S}{I\left(1+\alpha_{1} B\right)}-\frac{\beta_{2} \alpha_{2} S I}{\left(1+\alpha_{2} I\right)^{2}}-\mu_{1}\right)\|z\| .
$$

It is easy to see that (56) also holds for $U_{1}>U_{2}$ and $z_{2}, z_{3}<0<z_{1}$ when $\left|z_{1}\right|<\left|z_{2}\right|$, which can be obtained by linearity.

Case $3\left(U_{2}(z)>U_{1}(z)\right.$ and $\left.z_{6}<0<z_{4}, z_{5}\right)$. In this case, $\|z\|=\max \left\{\left|z_{4}\right|+\left|z_{5}\right|,\left|z_{4}\right|+\left|z_{6}\right|\right\}$.

Subcase $3.1\left(\left|z_{5}\right|>\left|z_{6}\right|\right)$. Then $\|z\|=\left|z_{4}\right|+\left|z_{5}\right|=z_{4}+z_{5}$ and $U_{1}(z)<\left|z_{4}\right|+\left|z_{5}\right|$. Taking the right-hand derivative of $\|z\|$, we obtain

$$
\begin{aligned}
D_{+}\|z\|= & z_{4}^{\prime}+z_{5}^{\prime}=\frac{\eta I}{B} z_{2} \\
& +\left(-\frac{\beta_{1} B}{1+\alpha_{1} B}-\frac{\beta_{2} I}{1+\alpha_{2} I}-\frac{\eta I}{B}-\mu_{1}-\phi\right) z_{4}+\theta z_{5} \\
& +\left(-\frac{\beta_{2} S}{\left(1+\alpha_{2} I\right)^{2}}\right) z_{6}+\frac{\eta I}{B} z_{3}+\phi z_{4} \\
& +\left(-\frac{\eta I}{B}-\mu_{1}-\theta\right) z_{5}
\end{aligned}
$$

$$
\begin{aligned}
\leq & \frac{\eta I}{B}\left(\left|z_{2}\right|+\left|z_{3}\right|\right) \\
& +\left(-\frac{\beta_{1} B}{1+\alpha_{1} B}-\frac{\beta_{2} I}{1+\alpha_{2} I}-\frac{\eta I}{B}-\mu_{1}\right)\left|z_{4}\right| \\
& +\left(-\frac{\eta I}{B}-\mu_{1}\right)\left|z_{5}\right|+\frac{\beta_{2} S}{\left(1+\alpha_{2} I\right)^{2}}\left|z_{6}\right| \\
\leq & \left(\frac{\beta_{2} S}{\left(1+\alpha_{2} I\right)^{2}}-\mu_{1}\right)\left(\left|z_{4}\right|+\left|z_{5}\right|\right) .
\end{aligned}
$$

Recalling that $\|z\|=\left|z_{4}\right|+\left|z_{5}\right|$ yields

$$
D_{+}\|z\| \leq\left(\frac{\beta_{2} S}{\left(1+\alpha_{2} I\right)^{2}}-\mu_{1}\right)\|z\| .
$$

It is easy to see that (58) also holds for $U_{2}>U_{1}$ and $z_{4}, z_{5}<$ $0<z_{6}$ when $\left|z_{5}\right|>\left|z_{6}\right|$, which can be obtained by linearity.

Subcase $3.2\left(\left|z_{5}\right|<\left|z_{6}\right|\right)$. Then $\|z\|=\left|z_{4}\right|+\left|z_{6}\right|=z_{4}-z_{6}$ and $U_{1}(z)<\left|z_{4}\right|+\left|z_{6}\right|$. Taking the right-hand derivative of $\|z\|$, we obtain

$$
\begin{aligned}
D_{+}\|z\|= & z_{4}^{\prime}-z_{6}^{\prime}=\frac{\eta I}{B} z_{2} \\
& +\left(-\frac{2 \beta_{1} B}{1+\alpha_{1} B}-\frac{2 \beta_{2} I}{1+\alpha_{2} I}-\frac{\eta I}{B}-\mu_{1}-\phi\right) z_{4} \\
& +\theta z_{5}-\left(\frac{2 \beta_{2} S}{\left(1+\alpha_{2} I\right)^{2}}-\frac{\eta I}{B}-\mu_{1}-d-\alpha\right) z_{6} \\
\leq & \frac{\eta I}{B}\left|z_{2}\right|+\left(-\frac{2 \beta_{1} B}{1+\alpha_{1} B}-\frac{2 \beta_{2} I}{1+\alpha_{2} I}-\frac{\eta I}{B}\right. \\
& +\theta\left|z_{5}\right|+\left(\frac{2 \beta_{2} S}{\left(1+\alpha_{2} I\right)^{2}}-\frac{\eta I}{B}-\mu_{1}-d-\alpha\right)\left|z_{4}\right| \\
\leq & \left(\frac{2 \beta_{2} S}{\left(1+\alpha_{2} I\right)^{2}}+\theta-\mu_{1}\right)\left(\left|z_{4}\right|+\left|z_{6}\right|\right) .
\end{aligned}
$$

Therefore,

$$
D_{+}\|z\| \leq\left(\frac{2 \beta_{2} S}{\left(1+\alpha_{2} I\right)^{2}}+\theta-\mu_{1}\right)\|z\| .
$$

It is easy to see that (60) also holds for $U_{2}>U_{1}$ and $z_{4}, z_{5}<$ $0<z_{6}$ when $\left|z_{6}\right|>\left|z_{5}\right|$, which can be obtained by linearity. 
Combing the results of the six cases presented here in (50)-(60), as well as the remaining 10 cases, we obtain the result

$$
\begin{aligned}
& D_{+}\|z\| \\
& \leq \max \left\{-\mu_{1}, d+\alpha-\mu_{1}-\theta, \frac{2 \beta_{2}\left(\mu_{1}+\theta\right)}{\mu_{1}+\theta+\phi}+\theta-\mu_{1}\right\}\|z\|,
\end{aligned}
$$

when $I \geq 0, S \leq\left(\mu_{1}+\theta\right) /\left(\mu_{1}+\theta+\phi\right)$. Hence, $\mu(\mathfrak{B}) \leq$ $\max \left\{-\mu_{1}, d+\alpha-\mu_{1}-\theta,\left(\left(2 \beta_{2}\left(\mu_{1}+\theta\right)\right) /\left(\mu_{1}+\theta+\phi\right)\right)+\theta-\mu_{1}\right\}$. Therefore, $\mu(\mathfrak{B}) \leq 0$ in int $\Omega$ if $d+\alpha \leq \mu_{1}+\theta$ and $\left(\left(2 \beta_{2}\left(\mu_{1}+\right.\right.\right.$ $\left.\theta)) /\left(\mu_{1}+\theta+\phi\right)\right)+\theta \leq \mu_{1}$. Thus, Theorem 14 is satisfied. From Corollary 13, we can obtain that all solutions intersect the interior of $\Omega$ and the endemic equilibrium $E^{*}$ is globally asymptotically stable, completing the proof of Theorem 11.

\section{Sensitivity Analysis of $\mathscr{R}_{v}$}

To facilitate the interpretation of the sensitivity of $\mathscr{R}_{v}$, we now present some numerical simulations by using the set of parameters values in Table 1 .

Now, we regard the vaccinated rate $\phi$ and the waning rate $\theta$ as the control parameters, while the other parameters are fixed. From Figures 3 and 4, the effects of various parameters, that is, $\phi$ and $\theta$ on the control reproduction number $\mathscr{R}_{v}$ have been shown. It is noted that as the parameter $\phi$ increases, $\mathscr{R}_{v}$ decreases and as $\theta$ decreases, $\mathscr{R}_{v}$ decreases. In fact, we can obtain the critical values of $\phi$ and $\theta$ that reduce $\mathscr{R}_{v}$ to 1 :

$$
\begin{gathered}
\phi=\frac{\left(\mu_{1}+\theta\right)\left(\mu_{2} \beta_{2}+\beta_{1} \eta\right)-\mu_{2}\left(\mu_{1}+\theta\right)\left(d+\alpha+\mu_{1}\right)}{\mu_{2}\left(d+\alpha+\mu_{1}\right)} \triangleq \phi_{v}, \\
\theta=\frac{\mu_{1}\left(\mu_{2} \beta_{2}+\beta_{1} \eta\right)-\mu_{2}\left(\mu_{1}+\phi\right)\left(d+\alpha+\mu_{1}\right)}{\left(\mu_{2} \beta_{2}+\beta_{1} \eta\right)-\mu_{2}\left(d+\alpha+\mu_{1}\right)} \triangleq \theta_{v} .
\end{gathered}
$$

In Figure 3, we select $\theta=0.07,0.03,0.007,0.0001$, corresponding $\phi_{v}=2.07,0.89,0.21,0.01$, respectively. We can see that when the wanning rate $\theta$ has a greater value, then there is no $\phi_{v}$ such that $\mathscr{R}_{v}<1$. Similarly, in Figure 4, we select $\phi=0.01,0.1,0.3,0.6,0.99$, corresponding $\theta_{v}=$ $0.0002,0.003,0.01,0.02,0.03$, respectively. We can see that when $\phi$ is smaller, then there is no $\theta_{v}$ such that $\mathscr{R}_{v}<1$. Thus, the control reproduction number cannot reduce below unity only by increasing $\theta$ or decreasing $\phi$. The critical values $\phi_{v}$ and $\theta_{v}$ are important in regulating the infection magnitude. In order to reduce $\mathscr{R}_{v}$ to 1 , a greater vaccinated rate than $\phi_{v}$ and a smaller wanning rate than $\theta_{v}$ have to be achieved simultaneously. We will deduce $\mathscr{R}_{v}$ below 1 by using both $\phi$ and $\theta$ at the same time, which can control cholera. Otherwise, the cholera persists (see Figure 5).

\section{Conclusion}

In this paper, we have conducted global stability analysis of a SVIR-B cholera model. Based on the imperfective vaccine, with the environment component incorporated and multiple

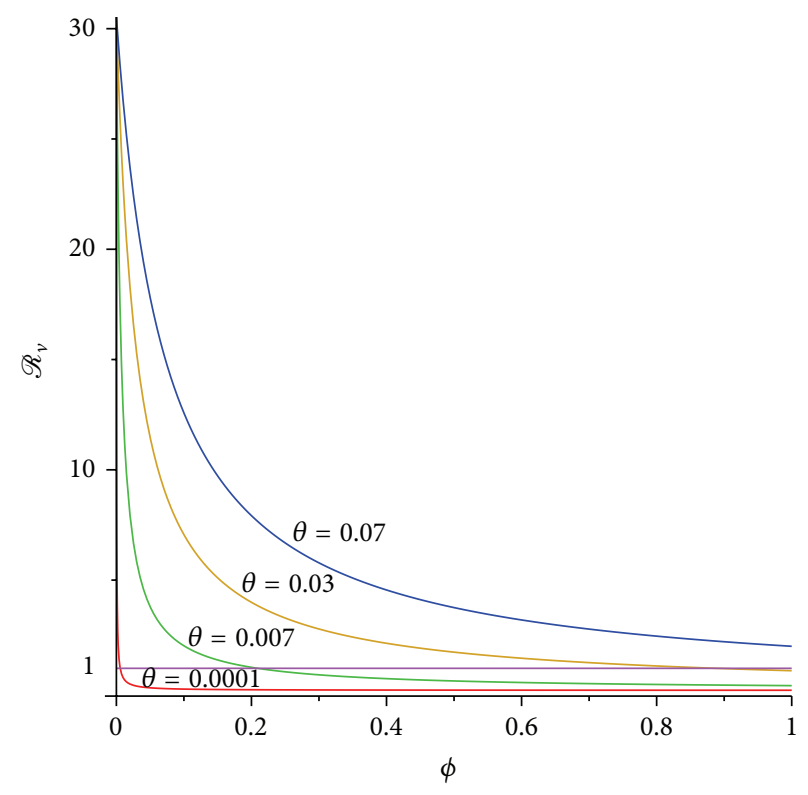

FIGURE 3: The contour diagram of the control reproduction number $\mathscr{R}_{v}$ with $\phi$ when $\theta$ has some fixed value.

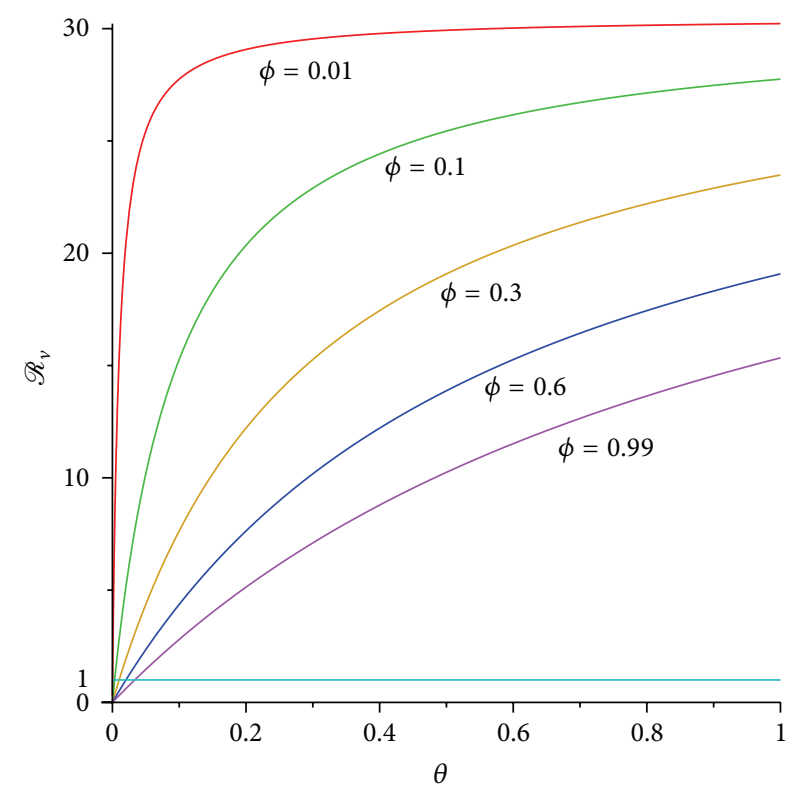

FIgURE 4: The contour diagram of the control reproduction number $\mathscr{R}_{v}$ with $\theta$ when $\phi$ has some fixed value.

transmission pathways coupled, the cholera models distinguish themselves from regular SIR epidemiological models. The mathematical results show that the control reproduction number $\mathscr{R}_{v}$ satisfies a threshold property with threshold value 1 . When $\mathscr{R}_{v}<1$, it has been proved that the diseasefree equilibrium $E_{0}$ is globally asymptotically stable under some sufficient conditions. And, when $\mathscr{R}_{v}>1$, the unique endemic equilibrium $E^{*}$ is globally asymptotically stable. This shows that cholera can be eliminated from the community if the imperfect vaccine brings $\mathscr{R}_{v}$ to a value less than unity. 
TABLE 1: Estimation of parameters.

\begin{tabular}{|c|c|c|c|}
\hline Parameters & Meaning & Values & Reference \\
\hline$\overline{\mu_{1}}$ & Natural human birth and death rate & $9.13 \times 10^{-5} /$ day & [3] \\
\hline$\beta_{1}$ & $\begin{array}{l}\text { Contact rates for the human-environment } \\
\text { interaction }\end{array}$ & $0.214 /$ day & [3] \\
\hline$\beta_{2}$ & $\begin{array}{l}\text { Contact rates for the human-human } \\
\text { interaction }\end{array}$ & $0.02 /$ day & [3] \\
\hline$d$ & Disease-induced death rate & $0.013 /$ day & {$[4]$} \\
\hline$\alpha$ & $\begin{array}{l}\text { Recovery rate at which people recover from } \\
\text { environment }\end{array}$ & $0.2 /$ day & [3] \\
\hline$\eta$ & $\begin{array}{l}\text { Contribution of infected individuals to the } \\
\text { population of Vibrio cholera }\end{array}$ & 10 & [7] \\
\hline$\mu_{2}$ & Net death rate of Vibrio cholera & $0.33 /$ day & [7] \\
\hline
\end{tabular}

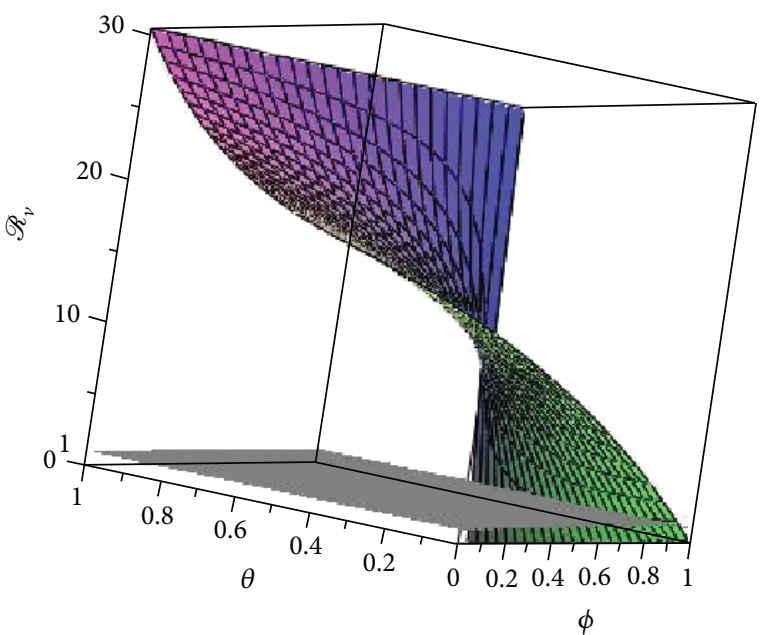

FIGURE 5: The contour diagram of the control reproduction number $\mathscr{R}_{v}$ with $\phi, \theta$ variables. All the other parameter values are the same as those in Figure 3.

Now, if we consider that the case there is no vaccination. Model (1) can be written as follows:

$$
\begin{gathered}
\frac{d S(t)}{d t}=\mu_{1}-\frac{\beta_{1} S(t) B(t)}{1+\alpha_{1} B(t)}-\frac{\beta_{2} S(t) I(t)}{1+\alpha_{2} I(t)}-\mu_{1} S(t), \\
\frac{d I(t)}{d t}=\frac{\beta_{1} S(t) B(t)}{1+\alpha_{1} B(t)}+\frac{\beta_{2} S(t) I(t)}{1+\alpha_{2} I(t)}-\left(d+\alpha+\mu_{1}\right) I(t), \\
\frac{d R(t)}{d t}=\alpha I(t)-\mu_{1} R(t), \\
\frac{d B(t)}{d t}=\eta I(t)-\mu_{2} B(t) .
\end{gathered}
$$

According to Theorem 2 in [31], the basic reproduction number of model $(*)$ is $\mathscr{R}_{0}=\left(\beta_{2} \mu_{2}+\beta_{1} \eta\right) /\left(\mu_{2}(d+\alpha+\right.$ $\left.\left.\mu_{1}\right)\right)$. We can express the control reproduction number $\mathscr{R}_{v}$ as $\mathscr{R}_{v}=\mathscr{R}_{0}\left(\left(\mu_{1}+\theta\right) /\left(\mu_{1}+\theta+\phi\right)\right)$. Note that $\mathscr{R}_{v} \leq \mathscr{R}_{0}$ with equality only if $\phi=0$. That is, despite being imperfect, the vaccine will always reduce the reproduction number of the disease, and in the absence of the vaccination, the disease transmission will be high. Further simulations in Section 7 also show that the vaccinated rate $(\phi)$ and the wanning rate $(\theta)$ play equal important roles in reducing the control reproduction number. The disease can be controlled if and only if the reproduction number is reduced to values less than unity if the vaccinated rate $(\phi)$ exceed the threshold $\phi_{v}$ and the wanning rate $(\theta)$ less than the threshold $\theta_{v}$ simultaneously. Therefore, vaccination is a good way to control cholera.

However, there are inherent disadvantages towards the vaccination modeling. For cholera with incubation period, it is hard to rapidly identify those with ambiguous symptoms [4]. Moreover, the vaccination does not always work well due to the limitations of medical development level and financial budget (some vaccines are very expensive and some portions of people cannot be covered) [32]. Nonetheless, [33] indicated that during cholera outbreaks (periodic control) vaccination campaigns can be a good strategy to control cholera epidemics. Besides, they pointed out that vaccination and improvement in the sanitation system and food/personal hygiene are the most efficient control strategies to prevent cholera transmission and outbreaks. Hence, incorporating some other control strategies, we may consider the more realistic ordinary differential equation model. The theoretical study of cholera models has been in progress and is an exciting area of future research.

\section{Appendices}

\section{A. The Proof of $a_{1} a_{2} a_{3}>a_{3}^{2}+a_{1}^{2} a_{4}$}

The proof of $a_{1} a_{2} a_{3}-a_{3}^{2}-a_{1}^{2} a_{4}=\xi_{1}^{3} \mu_{1}\left\{(\theta+\phi)^{2}+\mu_{1}(3 \theta+\right.$ $\left.\left.2 \mu_{1}+3 \phi\right)\right\}+\xi_{1}^{2}\left\{\left(4 \mu_{1}\left(\theta+\mu_{1}+\phi\right)+(\theta+\phi)^{2}+\xi_{2}+\mu_{1}(\theta+\phi)^{3}+\right.\right.$ $3 \mu_{1} \mu_{2}(\phi+\theta)^{2}+\mu_{1}^{2}\left(8 \mu_{1}+9 \mu_{2}\right)(\theta+\phi)+5 \mu_{1}^{2} \phi(\phi+2 \theta)+\mu_{1}^{2}\left(4 \mu_{1}^{2}+\right.$ $\left.\left.5 \theta^{2}+6 \mu_{1} \mu_{2}\right)\right\}+\xi_{1}\left\{\left(4 \mu_{1}^{3}+\mu_{1}\left(8 \mu_{2}+6 \mu_{1}\right)(\theta+\phi)+4 \mu_{1}(\theta+\phi)^{2}+\right.\right.$ $\left.\phi \mu_{2}(2 \phi+1+4 \theta)+(\theta+\phi)^{3}+\mu_{2}\left(2 \theta^{2}+8 \mu_{1}^{2}\right)\right) \xi_{2}+\left(\phi+\theta+2 \mu_{1}\right) \xi_{2}^{2}+$ $\mu_{1}^{2}\left(4 \mu_{1}+10 \mu_{2}\right)(\theta+\phi)^{2}+7 \theta \phi d \mu_{1}+\mu_{1} \mu_{2}\left(2 \theta^{3}+6 \theta^{2} \phi+2 \phi^{3}+5 d \phi\right)+$ $\mu_{1}^{3}\left(6 \mu_{2}^{2}+2 \mu_{1}^{2}\right)+3 \mu_{1} \mu_{2}^{2}\left(\theta^{2}+\phi^{2}\right)+\left(9 \mu_{1}^{2} \mu_{2}^{2}+16 \mu_{1}^{3} \mu_{2}+5 \mu_{1}^{4}\right)(\theta+$ 
$\left.\phi)+\mu_{1}^{2}\left(4 \theta^{2}+\phi^{3}+3 \theta \phi^{2}+\theta^{3}+8 \mu_{1}^{2} \mu_{2}+3 \theta^{2} \phi\right)\right\}+\xi_{2}^{2} \mu_{2}\left\{\phi+2 \mu_{1}+\right.$ $\theta\}+\xi_{2}\left\{\mu_{2}(\theta+\phi)^{3}+\mu_{2}\left(4 \mu_{1}+\mu_{2}\right)(\theta+\phi)^{2}+\mu_{1} \mu_{2}\left(4 \mu_{2}+6 \mu_{1}\right)(\theta+\right.$ $\left.\phi)+4 \mu_{1}^{2} \mu_{2}^{2}\right\}+\mu_{1} \mu_{2}\left(\mu_{1}+\mu_{2}\right)(\theta+\phi)^{3}+4 \mu_{1}^{3} \mu_{2}\left(4 \mu_{1}^{2}+5 \mu_{1} \mu_{2}+\right.$ $\left.\mu_{2}^{2}\right)(\theta+\phi)^{2}+\mu_{1}^{2} \mu_{2}\left(5 \mu_{1}^{2}+8 \mu_{1} \mu_{2}+3 \mu_{2}^{2}\right)(\theta+\phi)+\mu_{1}^{3} \mu_{2}^{2}\left(2 \mu_{2}+4 \mu_{1}\right)$.

From Section 4 , we know that $\xi_{1}>0, \xi_{2}>0$. After some algebraic manipulations, we have $a_{1} a_{2} a_{3}-a_{3}^{2}-a_{1}^{2} a_{4}>0$. Thus, $a_{1} a_{2} a_{3}>a_{3}^{2}+a_{1}^{2} a_{4}$, when $\mathscr{R}_{v}<1$.

\section{B. The Proof of $b_{1} b_{2} b_{3}>b_{3}^{2}+b_{1}^{2} b_{4}$}

The proof of $b_{1} b_{2} b_{3}-b_{3}^{2}-b_{1}^{2} b_{4}=\omega_{1}^{3} \mu_{1}\left\{(\theta+\phi)^{2}+3 \mu_{1}(\theta+\phi)+\right.$ $\left.2 \mu_{1}^{2}\right\}+\omega_{1}^{2}\left\{\omega_{2}\left((\theta+\phi)^{2}+4 \mu_{1}\left(\theta+\phi+\mu_{1}\right)\right)+\mu_{1} \mu_{2}\left(\left(4 \mu_{1}+\alpha+4 \theta+\right.\right.\right.$ $\left.3 \phi+d) J_{1}+3(\theta+\phi)^{2}+(6+9 \phi) \mu_{1}\right)+\mu_{1}(\theta+\phi)^{3}+(\theta+\alpha+d) \cdot \phi J_{1} \mu_{2}+$ $5 \mu_{1}^{2}(\theta+\phi)^{2}+9 \theta \mu_{1}^{2}\left(\mu_{2}+J_{1}\right)+(\phi+2 d) \phi J_{1} \mu_{1}+\theta^{2} J_{1}\left(\mu_{2}+3 \mu_{1}\right)+$ $\mu_{1}^{3}\left(4 \mu_{1}+8 \theta\right)+3 J_{1} \mu_{1}^{2}(d+\alpha+2 \phi)+\theta J_{1}\left(\theta+\phi+4 \mu_{1}\right)(d+\alpha)+4 \theta \phi \mu_{1}+$ $\left.6 J_{1} \mu_{1}^{3}+2 \alpha \phi \mu_{1} J_{1}+8 \phi \mu_{1}^{3}\right\}+\omega_{1} \cdot\left\{\omega_{2}^{2}\left(\phi+2 \mu_{1}+\theta\right)+\omega_{2}\left(4 \mu_{1}(\phi+\theta)^{2}+\right.\right.$ $4 \mu_{1} \mu_{2}\left(2 \mu_{1}+2 \theta+2 \phi+J_{1}\right)+\phi \mu_{2}\left(2 \phi+J_{1}+4 \theta\right)+6 \cdot \mu_{1}^{2}(\theta+\phi)+3 \theta \phi J_{1}+$ $2 \theta J_{1}\left(d+\alpha+\theta+\mu_{2}\right)+(3 \alpha+3 d+5 \phi) \mu_{1} J_{1}+J_{1} \phi(\phi+\alpha+d)+2 \theta^{2}$. $\left.\mu_{2}+8 \theta \mu_{1} J_{1}+(\theta+\phi)^{3}+4 \mu_{1}^{2}\left(2 J_{1}+\mu_{1}\right)+J_{1} \mu_{2}(d+\alpha)\right)+\mu_{1}\left(\mu_{2}+\mu_{1}+\right.$ $\left.2 J_{1}\right)(\theta+\phi)^{3}+\left(4 \cdot \mu_{1}^{3}+(d+\alpha+\theta) \mu_{2} J_{1}+\mu_{1} \mu_{2}\left(10 \mu_{1}+3 \mu_{2}\right)+\left(d \theta^{2}+\right.\right.$ $\left.\left.4 \mu_{1}^{2}+\alpha \theta\right) J_{1}\right)(\theta+\phi)^{2}+\mu_{1}^{2}\left(9 \mu_{2}^{2}+4 \mu_{1}^{2}\right)(\theta+\phi)+8 \mu_{1}^{4}\left(\mu_{2}+J_{1}\right)+2 \mu_{1}^{5}+$ $\mu_{1}^{3}\left(5 d+16 \theta+6 J_{1}+5 \alpha+11 \phi\right) J_{1}+3 \mu_{1}^{2} J_{1}\left(\left(2 \alpha J_{1}+6 d+\phi\right) J_{1}+2 \theta^{2}+\right.$ $\left.3 \theta\left(d+\alpha+J_{1}\right)+2(d+\alpha+\theta) \phi\right)+\mu_{1}\left(\left((\alpha+d)^{2}+8(d+\alpha) \theta+2(d+\alpha+\right.\right.$ $\left.\left.\theta) \phi+3 \theta^{2}\right) J_{1}^{2}+\left(3 d \theta^{2}+5 \alpha \theta^{2}+\phi(d+\alpha)(2 \phi+7 \theta)\right) J_{1}\right)+\mu_{1} \mu_{2}((5 \alpha+$ $\left.8 \theta+3 \phi+5 d) J_{1}^{2}+5\left((d+\alpha)(\theta+\phi)+3 \theta \phi+\phi^{2}\right) J_{1}\right)+\mu_{2}^{2}((d+\alpha+$ $\left.\theta)\left(J_{1}+2 \phi\right) J_{1}+2 \theta^{2} J_{1}\right)+\mu_{2} J_{1}^{2}\left((d+\theta)^{2}+(\alpha+\theta)^{2}+(d+\alpha+\theta) \phi+\right.$ $2 \alpha d)+2 \mu_{1} \mu_{2}^{2} J_{1}\left(d J_{1}+4 \theta+3 \phi+\alpha\right)+\mu_{1}^{2} \mu_{2}\left(8 J_{1}^{2}+16 \mu_{1}\left(\theta+\phi+J_{1}\right)+\right.$ $\left.\left(5 \alpha+5 d \mu_{1}+24 \theta+19 \phi\right) J_{1}\right)+\mu_{1}^{2} \mu_{2}^{2}\left(6 \mu_{1}+8 J_{1}\right)+J_{1}^{2}\left(\theta(\alpha+d)^{2}+\theta(d+\right.$ $\alpha)(2 \theta+\phi))\}+\omega_{2}^{2}\left\{\phi J_{1}+\theta \mu_{2}+2 \mu_{1} J_{1} \phi \mu_{2}+\theta J_{1}+2 \mu_{1} \mu_{2}\right\}+\omega_{2}\left\{\left(\mu_{2}(\theta+\right.\right.$ $\phi)^{3}+\left(2 \mu_{2} J_{1}+4 \mu_{1} \mu_{2}+\theta J_{1}\right)(\theta+\phi)^{2}+2 \mu_{1}^{2} J_{1}\left(3 \theta+d+\alpha+2 \mu_{1}+2 J_{1}+\right.$ $2 \phi)+\mu_{1}\left((4 \theta+3 \alpha+3 d+\phi) J_{1}^{2}+\left((\theta+\phi)^{2}+3 \phi(d+\alpha)+\theta(\phi+\alpha+\right.\right.$ d) $\left.) J_{1}\right)+\mu_{2}^{2}\left((\alpha+d+\phi+2 \theta) J_{1}+(\phi+2 \theta) \phi\right)+\mu_{1} \mu_{2} J_{1}((d+\alpha+8 \theta+7 \phi+$ $\left.\left.4 \cdot J_{1}\right)+4 \phi^{2}+8 \theta \phi\right)+\mu_{2} J_{1}\left((\alpha+d+\phi+2 \theta) J_{1}+(d+\alpha) \theta\right)+6 \mu_{1}^{2} \mu_{2}(\phi+$ $\theta)+4 \mu_{1} \mu_{2}^{2}\left(\phi+\theta+J_{1}+\mu_{1}\right)+(d \phi+d \theta+\alpha \phi) \phi J_{1}+((\alpha+d+\theta) \phi+(2 d+$ $\left.\theta+2 \alpha) \theta) J_{1}^{2}\right\}+\left(\mu_{1} \mu_{2}\left(2 \theta J_{1}+\mu_{2}^{2}+4 \mu_{1}^{2}+5 \mu_{1} \mu_{2}\right)+\theta J_{1} \mu_{2}^{2}\right)(\theta+\phi)^{2}+$ $\mu_{1} \mu_{2}\left(\mu_{1}^{2}+\mu_{2}\right)(\theta+\phi)^{3}+\mu_{1} \mu_{2}\left\{4 J_{1}^{3}(d+\alpha+\theta)+J_{1}^{2}(6(d+\alpha)(\theta+\phi)+\right.$ $\left.\left.(d+\alpha)^{2}+\theta(7 \theta+6 \phi)\right)+(d+\alpha)(\theta+\phi)(\theta+2 \phi) J_{1}\right\}+\mu_{1}^{3} \mu_{2}\left\{4 J_{1}^{3}+(7 \alpha+\right.$ $7 d+15 \theta+8 \cdot \phi) J_{1}^{2}+(5 \phi(\alpha+\phi+d)+(16+15 \phi+10 \theta+4 d) \theta) J_{1}+$ $4 \theta \alpha\}+\mu_{1}^{4}\left\{J_{1}\left(2 \alpha+2 \mu_{1}+5 \theta+3 \phi+2 \cdot d\right)+4 J_{1}^{2}\right\}+\mu_{1}^{3}\left\{2 J_{1}^{3}+J_{1}^{2}(5 d+5 \alpha+\right.$ $\left.3 \phi+8 \theta)+J_{1}\left(4 \theta^{2}+3 \phi(d+\alpha)+\phi^{2}+5 \theta(d+\alpha+\phi)\right)\right\}+\mu_{1}^{2}\left\{J_{1}^{2}\left(3 J_{1}+\right.\right.$ $4 \phi)(\theta+\alpha+d)+\left(9 \theta(\alpha+d)+(d+\alpha)^{2}+5 \theta^{2}\right)+\left(\theta\left(\theta^{2}+2 \phi \theta+5 d \phi+\right.\right.$ $\left.5 \alpha \phi)\}+4 \cdot \theta^{2}(\alpha+d)\right) J_{1}+\phi^{2} J_{1}(d+\theta+\alpha)+\mu_{1}\{(\theta(4 d+\theta+4 \alpha)+$ $\left.(\alpha+d)^{2}\right) J_{1}^{3}+\left(\theta(d+\theta)^{2}+\alpha(\theta+\phi)(\alpha+2 d)+(\phi+3 d+5 \alpha) \theta^{2}+\right.$ $\left.\left.5 \phi \theta(d+\alpha)+\phi d^{2}\right) J_{1}^{2}+\left(\theta\left(\phi^{2}+\theta^{2}\right)(d+\alpha)+2 \phi \theta^{2} \alpha\right) J_{1}\right\}+\mu_{2}^{3}\left\{J_{1}^{2}(\alpha+\right.$ $\left.\theta+d)+J_{1}\left(\theta^{2}+\phi(d+\alpha+\theta)\right)\right\}+\mu_{2}^{2}\left\{J_{1}^{3}(\alpha+d+\theta)+J_{1}^{2}((2 \phi+\theta)(\theta+\right.$ $\left.\alpha+d)+\theta)+J_{1} \phi(d+\alpha)(\theta+\phi)\right\}+\mu_{2}\left\{J_{1}^{3}\left((\theta+\alpha)^{2}+2 d(\alpha+\theta)+d^{2}\right)+\right.$ $\left.J_{1}^{2}\left(\theta^{2} d+(\alpha+\theta) \theta^{2}+\phi(d+\theta)^{2}+\phi \alpha(\alpha+2 \theta+2 d)\right)\right\}+\mu_{1} \mu_{2}^{2}\left\{2 J_{1}^{3}+\right.$ $\left.J_{1}^{2}(3 \alpha+3 d+5 \phi+8 \theta)+J_{1}\left(7 \theta^{2}+11 \theta \phi+4 \phi^{2}+(\theta+3 \phi)(d+\alpha)\right)\right\}+$ $\mu_{1} \mu_{2}^{3}\left(J_{1}\left(d+3 \phi+4 \theta+\alpha+2 J_{1}\right)\right)+\mu_{1}^{3} \mu_{2}\left(10 J_{1}^{2}+J_{1}(13 \phi+3 \alpha+\right.$ $3 d))+\mu_{1}^{2} \mu_{2}^{2}\left\{J_{1}\left(8 J_{1}+2 d+4 \mu_{2}+10 \mu_{1}+13 \phi+2 \alpha+15 \theta\right)+\left(3 \mu_{2}+\right.\right.$ $\left.\left.8 \mu_{1}\right)(\theta+\phi)+4 \mu_{1}^{2}+2 \mu_{1} \mu_{2}\right\}+\mu_{1}^{4} \mu_{2}\left\{8 J_{1}+5 \phi+5 \theta+2 \mu_{1}\right\}+J_{1}^{3}\{\theta(\alpha+$ $\left.d)^{2}+\theta^{2} \alpha\right\}+J_{1}^{2}\left\{\theta \phi d^{2}+\theta^{2}(d+\alpha)(\theta+\phi)\right\}+2 d \theta \phi\left(\alpha+\theta J_{1}\right)$.

From Section 6, we know that $\omega_{1}>0, \omega_{2}>0$. After some algebraic manipulations, we have $b_{1} b_{2} b_{3}-b_{3}^{2}-b_{1}^{2} b_{4}>0$. Thus, $b_{1} b_{2} b_{3}>b_{3}^{2}+b_{1}^{2} b_{4}$, when $\mathscr{R}_{v}>1$.

\section{The Second Additive Compound Matrix}

If $A=\left[a_{i j}\right]$ is an $n \times n$ matrix, its second additive compound $A^{[2]}$ is $\left(\begin{array}{c}n \\ 2\end{array}\right) \times\left(\begin{array}{c}n \\ 2\end{array}\right)$ matrix defined as follows. For any integer $i=1, \ldots,\left(\begin{array}{c}n \\ 2\end{array}\right)$, let $(i)=\left(i_{1}, i_{2}\right)$ such that $1 \leq i_{1}<i_{2} \leq n$. Then the element in the $i$-row and $j$-column of $A^{[2]}$ is

$$
a_{i_{1} i_{1}}+a_{i_{2} i_{2}}, \text { if }(j)=(i),
$$

$(-1)^{r+s} a_{i_{r} i_{s}}$, if exactly one entry $i_{r}$ of $(i)$

does not occur in $(j)$ and $j_{s}$

does not occur in $(i)$,

0 , if neither entry from $(i)$ occurs in $(j)$.

In the special case when $n=2$, we have $A_{2 \times 2}^{[2]}=a_{11}+a_{22}=$ $\operatorname{tr} A$. In general, each entry of $A^{[2]}$ is a linear expression of those of $A$. For instance, when $n=3$, the second additive compound matrix of $A=\left(a_{i j}\right)$ is

$$
A^{[2]}=\left(\begin{array}{ccc}
a_{11}+a_{22} & a_{23} & -a_{13} \\
a_{32} & a_{11}+a_{33} & a_{12} \\
-a_{31} & a_{21} & a_{22}+a_{33}
\end{array}\right),
$$

when $n=4$, the second additive compound matrix of $A=$ $\left(a_{i j}\right)$ is

$$
A^{[2]}=\left(\begin{array}{cccccc}
a_{11}+a_{22} & a_{23} & a_{24} & -a_{13} & -a_{14} & 0 \\
a_{32} & a_{11}+a_{33} & a_{34} & a_{12} & 0 & -a_{14} \\
a_{42} & a_{43} & a_{11}+a_{44} & 0 & a_{12} & a_{13} \\
-a_{31} & a_{21} & 0 & a_{22}+a_{33} & a_{34} & -a_{24} \\
-a_{41} & 0 & a_{21} & a_{43} & a_{22}+a_{44} & a_{23} \\
0 & -a_{41} & a_{31} & -a_{42} & a_{32} & a_{33}+a_{44}
\end{array}\right) .
$$


For detailed discussions of compound matrices and their properties, readers can refer to [34, 35]. A comprehension survey on compound matrices and their relations to differential equations is given in [34].

\section{Acknowledgments}

This work is supported by the National Natural Science Foundation of China (no. 11071011), Funding Project for Academic Human Resources Development in Institutions of Higher Learning under the Jurisdiction of Beijing Municipality (no. PHR201107123), and Basic and Frontier Technology Research Program of Henan Province (nos. 132300410025 and 1323000410364).

\section{Conflict of Interests}

The authors declare that they have no financial and personal relationships with other people or organizations that can inappropriately influence their work. There is no professional or other personal interest of any nature or kind in any product, service, or company that could be construed as influencing the position presented in or the review of the paper.

\section{References}

[1] A. K. T. Kirschner, J. Schlesinger, A. H. Farnleitner et al., "Rapid growth of planktonic Vibrio cholerae non-O1/non-O139 strains in a large alkaline lake in Austria: Dependence on temperature and dissolved organic carbon quality," Applied and Environmental Microbiology, vol. 74, no. 7, pp. 2004-2015, 2008.

[2] J. Reidl and K. E. Klose, "Vibrio cholerae and cholera: out of the water and into the host," FEMS Microbiology Reviews, vol. 26, no. 2, pp. 125-139, 2002.

[3] D. M. Hartley, J. G. Morris Jr., and D. L. Smith, "Hyperinfectivity: a critical element in the ability of V. cholerae to cause epidemics?" PLoS Medicine, vol. 3, no. 1, pp. 63-69, 2006.

[4] World Health Organization, 2012, http://www.who.int/gho/ epidemic_diseases/cholera/en/index.

[5] V. Capasso and G. Serio, "A generalization of the KermackMcKendrick deterministic epidemic model," Mathematical Biosciences, vol. 42, no. 1-2, pp. 43-61, 1978.

[6] V. Capasso and S. L. Paveri-Fontana, "Mathematical model for the 1973 cholera epidemic in the European Mediterranean region," Revue d'Epidemiologie et de Sante Publique, vol. 27, no. 2, pp. 121-132, 1979.

[7] C. T. Codeço, "Endemic and epidemic dynamics of cholera: the role of the aquatic reservoir," BMC Infectious Diseases, vol. 1, article 1, 2001.

[8] Z. Mukandavire, S. Liao, J. Wang, H. Gaff, D. L. Smith, and J. G. Morris Jr., "Estimating the reproductive numbers for the 2008-2009 cholera outbreaks in Zimbabwe," Proceedings of the National Academy of Sciences of the United States of America, vol. 108, no. 21, pp. 8767-8772, 2011.

[9] S. Liao and J. Wang, "Global stability analysis of epidemiological models based on Volterra-Lyapunov stable matrices," Chaos, Solitons and Fractals, vol. 45, no. 7, pp. 966-977, 2012.
[10] F. Fenner, D. A. Henderson, I. Arita, Z. Jezek, and I. D. Ladnyi, "Smallpox and its eradition," Journal of Community Health, vol. 13, no. 4, pp. 245-592, 1988.

[11] H. W. Hethcote, "Oscillations in an endemic model for pertussis," The Canadian Applied Mathematics Quarterly, vol. 6, no. 1, pp. 61-88, 1998.

[12] H. W. Hethcote, "The mathematics of infectious diseases," SIAM Review, vol. 42, no. 4, pp. 599-653, 2000.

[13] X. H. Tian, "Stability analysis of a delayed SIRS epidemic model with vaccination and nonlinear incidence," International Journal of Biomathematics, vol. 5, no. 6, 18 pages, 2012.

[14] M.-L. Garly and P. Aaby, "The challenge of improving the efficacy of measles vaccine," Acta Tropica, vol. 85, no. 1, pp. 117, 2003.

[15] J. Arino, C. C. Mccluskey, and P. V. Van Den Driessche, "Global results for an epidemic model with vaccination that exhibits backward bifurcation," SIAM Journal on Applied Mathematics, vol. 64, no. 1, pp. 260-276, 2003.

[16] Y. Kang and H. Z. Zhang, "The study status of oral cholera vaccine," Chinese Frontier Health Quarantine, vol. 28, no. sl, pp. 91-92, 2005.

[17] S. Singh, P. Chandra, and J. B. Shukla, "Modeling and analysis of the spread of carrier dependent infectious diseases with environmental effects," Journal of Biological Systems, vol. 11, no. 3, pp. 325-335, 2003.

[18] X. Yang, L. Chen, and J. Chen, "Permanence and positive periodic solution for the single-species nonautonomous delay diffusive models," Computers and Mathematics with Applications, vol. 32, no. 4, pp. 109-116, 1996.

[19] C. Castillo-Chavez, Z. Feng, and W. Huang, "On the computation of $R_{0}$ and its role on global stability," in Mathematical Approaches for Emerging and Reemerging Infectious Diseases: An introduction, vol. 65 of The IMA Volumes in Mathematics and Its Applications, pp. 229-250, Springer, New York, NY, USA, 2003.

[20] D. Mukherjee, "Uniform persistence in a generalized preypredator system with parasitic infection," BioSystems, vol. 47, no. 3, pp. 149-155, 1998.

[21] H. I. Freedman and P. Waltman, "Persistence in a model of three competitive populations," Mathematical Biosciences, vol. 73, no. 1, pp. 89-101, 1985.

[22] V. Hutson and G. T. Vickers, "A criterion for permanent coexistence of species, with an application to a two-prey onepredator system," Mathematical Biosciences, vol. 63, no. 2, pp. 253-269, 1983.

[23] H. I. Freedman, S. Ruan, and M. Tang, "Uniform persistence and flows near a closed positively invariant set," Journal of Dynamics and Differential Equations, vol. 6, no. 4, pp. 583-600, 1994.

[24] G. Butler and P. Waltman, "Persistence in dynamical systems," Journal of Differential Equations, vol. 63, no. 2, pp. 255-263, 1986.

[25] R. A. Smith, "Some applications of Hausdorff dimension inequalities for ordinary differential equations," Proceedings of the Royal Society of Edinburgh Section A, vol. 104, no. 3-4, pp. 235-259, 1986.

[26] M. Y. Li and J. S. Muldowney, "A geometric approach to globalstability problems," SIAM Journal on Mathematical Analysis, vol. 27, no. 4, pp. 1070-1083, 1996.

[27] M. Y. Li and J. S. Muldowney, "On Bendixson's criterion," Journal of Differential Equations, vol. 106, no. 1, pp. 27-39, 1993. 
[28] M. Y. Li and J. S. Muldowney, “On R. A. Smith's autonomous convergence theorem," The Rocky Mountain Journal of Mathematics, vol. 25, no. 1, pp. 365-378, 1995.

[29] R. H. Martin Jr., "Logarithmic norms and projections applied to linear differential systems," Journal of Mathematical Analysis and Applications, vol. 45, no. 2, pp. 432-454, 1974.

[30] A. B. Gumel, C. C. McCluskey, and J. Watmough, "An sveir model for assessing potential impact of an imperfect anti-SARS vaccine," Mathematical Biosciences and Engineering, vol. 3, no. 3, pp. 485-512, 2006.

[31] P. Van Den Driessche and J. Watmough, "Reproduction numbers and sub-threshold endemic equilibria for compartmental models of disease transmission," Mathematical Biosciences, vol. 180, pp. 29-48, 2002.

[32] M. Chen, "The opinion of WHO about cholera vaccine," International Journal of Biologicals, vol. 34, no. 4, pp. 214-218, 2011.

[33] R. P. Sanches, C. P. Ferreira, and R. A. Kraenkel, "The role of immunity and seasonality in cholera epidemics," Bulletin of Mathematical Biology, vol. 73, no. 12, pp. 2916-2931, 2011.

[34] J.S. Muldowney, "Compound matrices and ordinary differential equations," The Rocky Mountain Journal of Mathematics, vol. 20, no. 4, pp. 857-872, 1990.

[35] M. Fiedler, "Additive compound matrices and an inequality for eigenvalues of symmetric stochastic matrices," Czechoslovak Mathematical Journal, vol. 24, no. 99, pp. 392-402, 1974. 


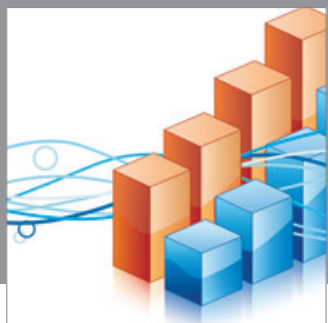

Advances in

Operations Research

mansans

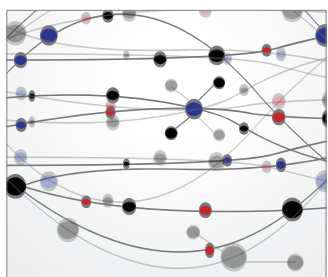

The Scientific World Journal
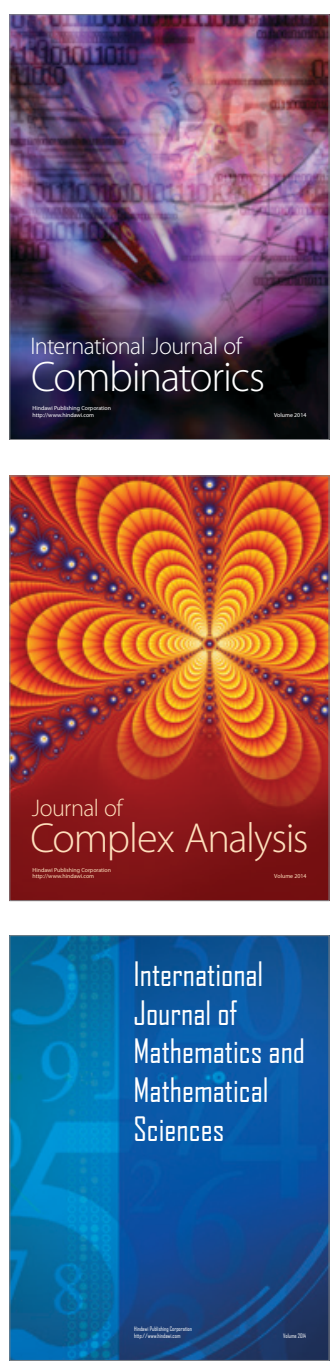
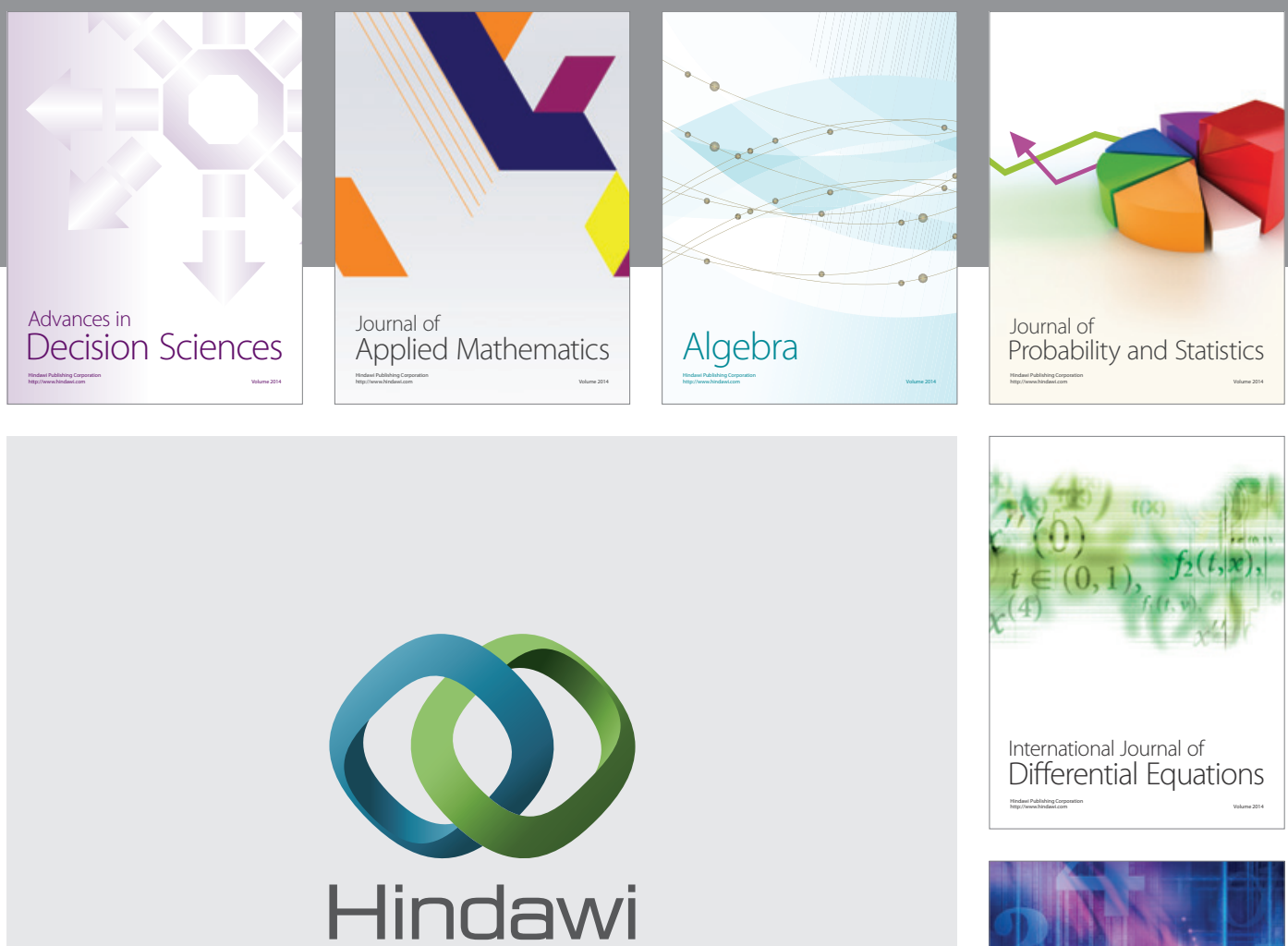

Submit your manuscripts at http://www.hindawi.com
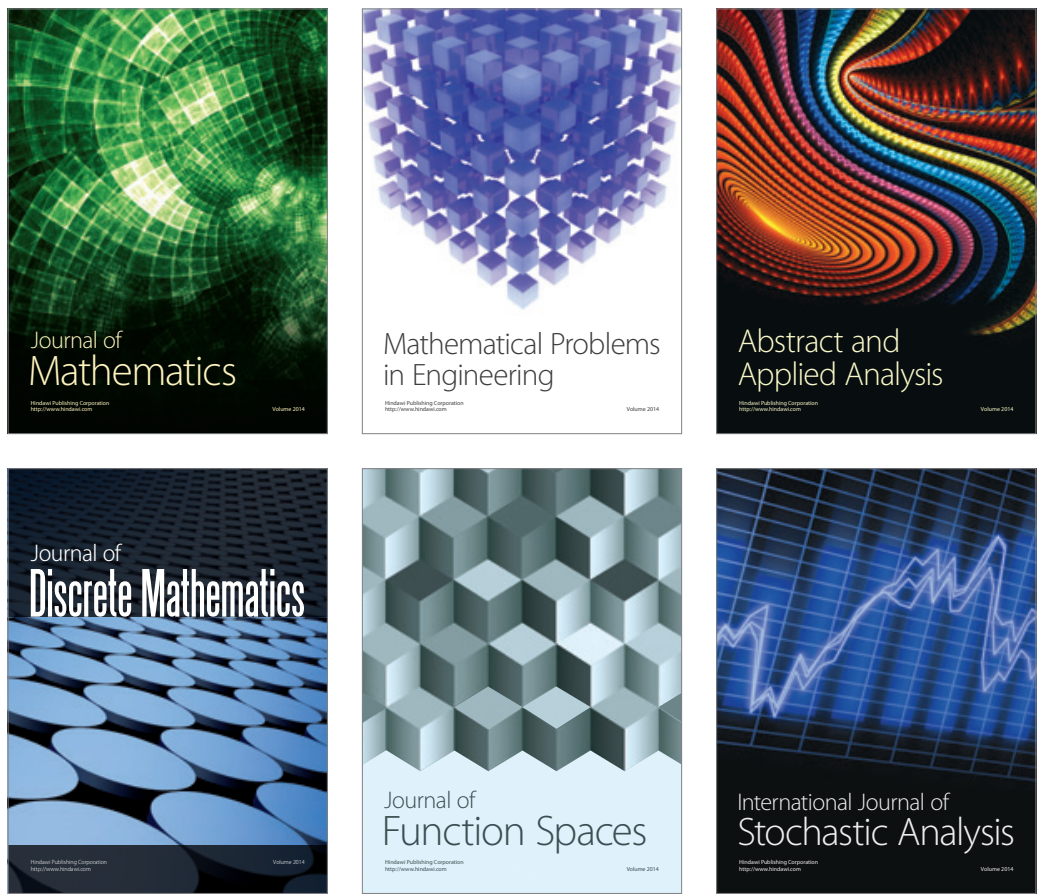

Journal of

Function Spaces

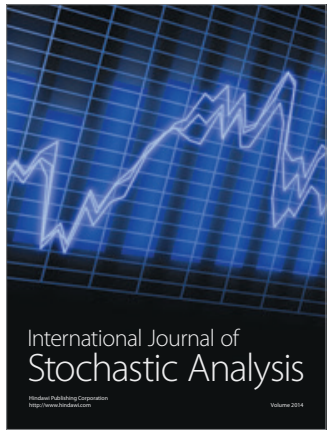

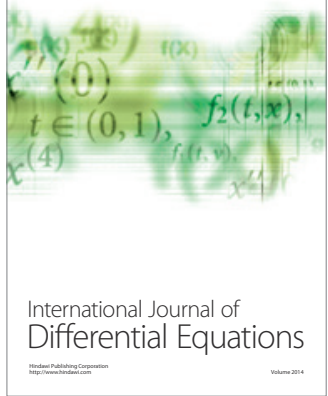
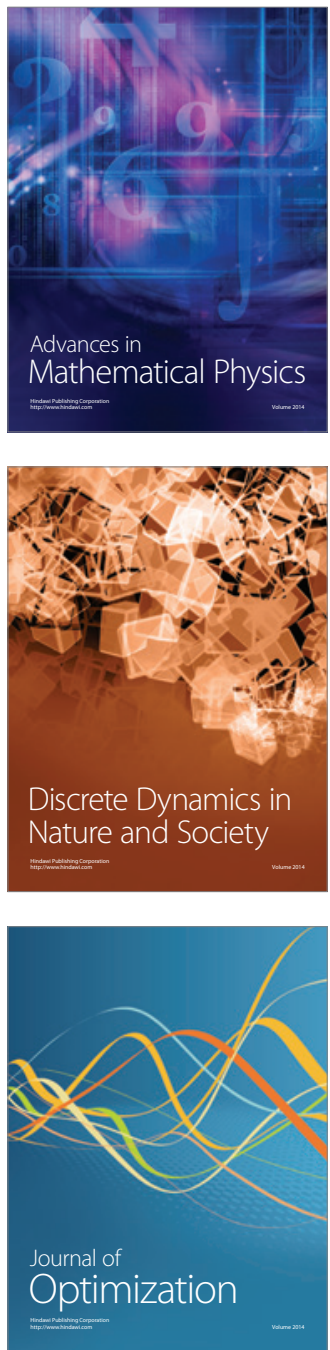